\title{
Structure of colloid-polymer suspensions
}

\author{
Matthias Fuchs ${ }^{1,3}$ and Kenneth S Schweizer ${ }^{2}$ \\ ${ }^{1}$ Department of Physics and Astronomy, The University of Edinburgh, JCMB King's Buildings, \\ Edinburgh EH9 3JZ, UK \\ ${ }^{2}$ Departments of Materials Science and Engineering and Chemistry, and Materials Research \\ Laboratory, University of Illinois, Urbana, IL 61801, USA
}

Received 12 October 2001, in final form 19 February 2002

Published 15 March 2002

Online at stacks.iop.org/JPhysCM/14/R239

\begin{abstract}
We discuss structural correlations in mixtures of free polymer and colloidal particles on the basis of a microscopic, two-component liquid-state integral equation theory. Whereas in the case of polymers much smaller than the spherical particles the relevant polymer degree of freedom is the centre of mass, for polymers larger than the (nano-) particles, conformational rearrangements need to be considered. They have the important consequence that the polymer depletion layer exhibits two widely different length scales, one of the order of the particle radius, the other of the order of the polymer radius or the polymerdensity screening length in dilute or semidilute concentrations, respectively. Because we find a spinodal instability (mostly) below the overlap concentration, the latter length is (mostly) set by the radius of gyration. As a consequence of the structure of the depletion layer, the particle-particle correlations depend on both length scales for large polymers. Because of the high local compressibility of large polymers, the local depletion layer is a strong function of particle density, but a weak function of polymer concentration. The amplitude of the long-ranged tail of the depletion layer also depends asymptotically only on the colloid concentration, while the range increases upon approaching the (meanfield) spinodal. The colloid correlations may be understood as characteristic for particles with a short-ranged potential when small polymers are added, and as characteristic for particles with a long-ranged, van der Waals-like attraction when the added free polymer coils are much larger. Small polymers fill the voids between the particles rather homogeneously, exhibiting correlations inside the mesh (which gets squeezed by the colloids) and Porod-like correlations for larger distances. The structure factor of large polymers, however, exhibits no ramified mesh and becomes a Lorentzian characterized by the mixture correlation length, which diverges at the spinodal.
\end{abstract}

${ }^{3}$ Permanent address: Physik-Department, Technische Universität München, 85747 Garching, Germany.

Konstanzer Online-Publikations-System (KOPS)

URL: http://www.ub.uni-konstanz.de/kops/volltexte/2007/3779/

URN: http://nbn-resolving.de/urn:nbn:de:bsz:352-opus-37798 


\section{Introduction}

Polymer-colloid suspensions are generally three-component systems composed of solid or impenetrable particles, macromolecules, and solvent [1-3]. The latter are usually small molecules compared to the polymer coils and colloids, and thus solvent is generally not explicitly treated by theories but rather taken as a background continuum which can influence the effective interactions between the other two components. The strong geometric asymmetry between polymers and particles is at the heart of their novel physical behaviour and useful material properties. All size asymmetry regimes are of importance, spanning the range from the traditional problem of large colloids and small polymers, to small nanoparticles and large macromolecules. The statistical geometry of polymers can be highly variable, including structures such as ideal random coils, self-avoiding random walks, semiflexible persistent chains, star-branched macromolecules, rigid rods, and fractal aggregates. The steric interactions between polymers, and polymers and colloids, depend on macromolecular geometry. Moreover, if the polymers are sufficiently flexible, the presence of colloids can potentially perturb their statistical conformation.

Great diversity also characterizes the particles spanning the range from the most common spherical geometry, to aspherical viruses, to highly anisotropic colloidal rods and plate- or discotic-type structures. The particles can be organic or inorganic solids, dendrimers, surfactant micelles, globular proteins, vesicles, or soft cross-linked microgels. Although the surfaces of particles are often homogeneous, they need not be. For example, both synthetic dendrimers and natural proteins possess chemically heterogeneous, or patchy, surfaces. Repulsive excludedvolume forces are universally present, and the geometric diversity provides a rich set of possibilities for packing in such suspensions. Of course, other forces are generally present and can be rationally tuned including van der Waals attractions, Coulombic forces, and specific interactions such as the hydrophobic effect [1-3].

There are also diverse mixture composition regimes which often are of interest to different scientific and engineering communities. In colloid or nanoparticle science, polymers are often used as low-concentration additives to manipulate the colloidal suspension properties [1-3]. On the other hand, in polymer science, solid particles are commonly employed as fillers to manipulate the properties of dense polymer melts, glasses, or rubbers [4], or as additives to modify the behaviour of polymer solutions which can form soft gels [5]. True composite materials where the volume fractions of particles and polymers are comparable are of significant scientific and applications interest.

In the present review, we focus on the simplest realization of these systems: hard spheres and flexible polymer chains under athermal 'good'-solvent conditions. This is the most fundamental and generic system characterized solely by hard-core repulsive interactions between all species. The statistical mechanical problem is a purely entropic 'packing problem'. For dilute and semidilute polymer concentrations [6], there are only three fundamental dimensionless variables: (i) the colloid packing fraction, $\phi_{c}$, (ii) the polymer-monomer or segment concentration reduced by its value at the dilute-semidilute crossover (where polymerpolymer interactions in a particle-free solution become important), $c / c^{*}$, and (iii) the size asymmetry, $R_{g} / R$, where $R$ is the colloid radius and $R_{g}$ the polymer radius of gyration. Despite the simple nature of the model, the physical behaviour of such mixtures is rich and complex [1-3,7-15]. In equilibrium the homogeneous fluid phase competes with fluidfluid and fluid-crystal phase-separated states. Non-equilibrium glass or gel states also play prominent roles in the experimental behaviour. Despite several decades of theoretical effort, it remains a major challenge to achieve a fundamental and predictive understanding within a microscopic framework of the thermodynamics, structure, and dynamic properties of the 
homogeneous fluid phase, and the equilibrium and non-equilibrium phase transitions, over the wide parameter range of experimental relevance.

A primary focus of this article is the structure and scattering patterns of athermal colloidpolymer mixtures. This is one of the most poorly understood aspects of these systems, and little information is available from experiment, theory, or computer simulation. The only theoretical approach currently available that can treat structural correlations of all species at the molecular level is the liquid-state integral equation methods. Thus, we shall concentrate on our recent contributions $[16,17]$ in this area based on the polymer reference interaction site model (PRISM) approach [18-22]. We first summarize the current level of theoretical understanding to place our work in its proper context.

The earliest theoretical model of athermal polymer-colloid suspensions was developed by Asakura and Oosawa (AO) [23]. They addressed the most elementary question of the effective entropy-driven 'depletion attraction' between two hard spheres dissolved in a polymer solution. Several major simplifications were introduced: (i) $R \gg R_{g}$, (ii) polymer coils were treated as hard spheres in their interactions with the large colloids, thereby ignoring internal conformational degrees of freedom, and (iii) polymer-polymer interactions and correlations were ignored and hence the polymer solution was effectively an ideal gas. Point (iii) is appropriate when $c \ll c^{*}$, or $c<c^{*}$ if the polymers are in special $\Theta$ solvents where the polymer-polymer second virial coefficient vanishes. The physical origin of the attraction can be viewed in two distinct, but equivalent, manners. When colloidal surfaces approach to less than a separation $\sim 2 R_{g}$, the polymer is 'squeezed out' of the gap between the particles resulting in an unbalanced polymer osmotic pressure exerted on the colloids, thereby pushing them together. Alternatively, the polymer (treated as a sphere with no internal structure) can increase its (translational) entropy if the particles cluster and share the excluded-volume depletion layer surrounding each of them.

For the above restrictive AO conditions, the finite colloid concentration problem can be approximately treated by adopting an effective one-component-fluid model where the polymers enter implicitly via an (assumed) pair-decomposable AO depletion potential. If one accepts the AO simplifications (i)-(iii), then three-body and higher effective interactions can be ignored if $R_{g} / R \leqslant 0.154$. Considerable progress in understanding the competition between fluid-fluid and fluid-crystal phase transitions was made using this approach by Gast et al [24]. However, as recently emphasized by Dijkstra et al [25], besides the obvious limitations, effective onecomponent models cannot be rigorously derived from the true binary mixture with interacting polymers since no small parameter exists even in the classic $R_{g} \ll R$ regime.

In the opposite limit to the AO model, when the colloidal particles can be considered as small point disturbances in the much larger polymer coils, integrating out the polymer degrees of freedom in the presence of few particles is tractable [26] and has led to deep insights [27-29]. This is possible because such point disturbances can be treated perturbatively; for randomwalk coils the change in free energy caused by one particle of size $R$ is of order $R R_{g}^{2} c \ll 1$. Whether this knowledge will lead to a theory for the particle structure remains open, because polymer-induced many-body interactions among the nanoparticles appear crucial.

If the polymers are treated as literally non-interacting ideal-random-walk coils, and polymer concentration is assumed to be vanishingly small, then a tractable two-component statistical thermodynamic theory can be constructed within the simplified AO model framework [30]. It is formally equivalent to the Widom-Rowlinson non-additive hard-sphere mixture problem [31], and the 'phantom-sphere free-volume' theory of Lekkerkerker et al [30] has had additional successes in qualitatively predicting polymer partitioning and three-phase equilibria in certain system parameter regimes. However, this approach has been recently shown experimentally to incur significant quantitative and qualitative errors for thermodynamic prop- 


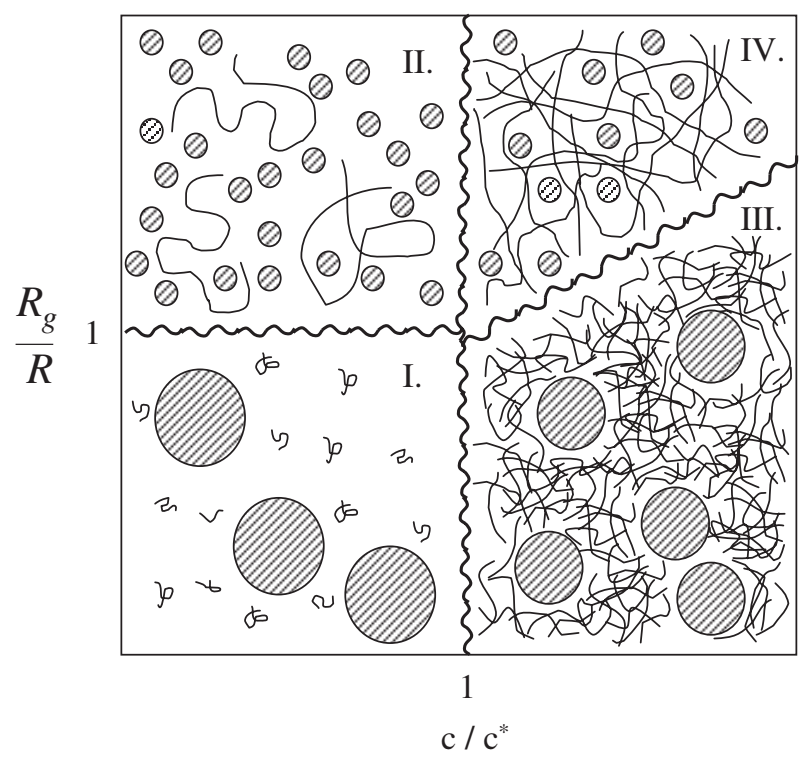

Figure 1. A schematic illustration of the four distinct physical regimes. Dilute and semidilute polymer solution regimes are divided according to $R_{g} \ll R$ (I) or $R_{g} \gg R$ (II), and $\xi \ll R$ (III) or $\xi \gg R(\mathrm{IV})$, respectively.

erties and fluid-fluid phase diagrams even when $R_{g}<R[32,33]$. Moreover, it is inappropriate for higher polymer concentrations $c>c^{*}$, and/or smaller particles where $R_{g}>R$.

Indeed, it is not clear what one means by 'depletion' if $R_{g} \gg R$ and polymers can 'wrap around' particles (see figure 1). Besides the obvious fact that pair-decomposable effective colloidal potentials lose meaning, interpretation in terms of 'squeezing polymer out' between surfaces and unbalanced osmotic pressure felt by colloids seems inapplicable. However, the fundamental idea that the perturbation of polymer coils by hard particles can be reduced, and polymer conformational entropy enhanced, by the physical clustering of particles remains valid. However, proper theoretical description now demands confronting the internal conformational degrees of freedom of the polymer coils, and how entropy loss due to excluded-volume interactions with colloids depends on particle-particle separation.

Thus, we believe an essential aim is that of constructing a microscopic two-component theory which can address within a common framework the four different physical regimes shown schematically in figure 1 . This requires treating polymers realistically as connected chains of units (monomers or segments) which experience excluded-volume forces among themselves, and with particles, at the elementary segmental scale. The role of polymer internal (conformational) degrees of freedom, or monomer density fluctuations, would then be directly taken into account, and its consequences for non-ideal polymer solution behaviour, including physical mesh formation at $c>c^{*}$, would emerge naturally. Moreover, no pair-decomposable depletion potential approximation would be necessary, particle penetration of the polymer coil would be accounted for, and segment-segment and segment-colloid spatial correlations could be predicted for the first time. A general goal of our recent work has been the formulation of such a theoretical approach that can give a unified description of thermodynamic properties, phase behaviour, and structure. Knowledge of the latter is of intrinsic interest, and also provides essential input into modern microscopic theories of the dynamics of such suspensions and their gelation or vitrification [34-38]. 
To achieve the above goals requires combining statistical mechanical ideas from the fields of polymer and colloid physics. The great range of relevant length scales, and geometric asymmetries between the species, have rendered computer simulation of polymer-colloid mixtures at this level nearly impossible [39-41]. An emerging attempt at a two-component description which approximates polymer chains as 'soft colloids' is interesting [42, 43]. However, such an approach avoids the explicit treatment of polymer internal degrees of freedom, is not applicable if $R_{g}>R$, and the subtle question of what effective polymer-colloid pair potential to employ has not yet been addressed. We have chosen to pursue a microscopic liquid-state theory approach based on atomic and macromolecular integral equation methods, in particular the PRISM theory [19] which is the polymeric generalization of the RISM theory of Chandler and Andersen [20,21] developed for small, rigid molecular fluids. The connection between polymer integral equation theory and (coarse-grained) Hamiltonian-based Gaussian field theory with constraints has been established [22]. The PRISM approach has been extensively developed and widely applied over the past decade [19] including the successful treatment of flexible and semiflexible polymer solutions, melts, alloys, self-assembling block copolymers, liquid-crystalline polymers [44], and star-branched macromolecules [45].

There are at least two major challenges to formulating a reliable, computationally convenient, liquid-state theory of athermal polymer-colloid suspensions. First, a fully numerically based approach which works at the segment level encounters severe difficulties spanning the 3-4 orders of magnitude of length scales (if $R \gg R_{g}$ ) from segment to colloid diameter [46]. Moreover, local chemical detail on the segmental scale may not be important for mesoscopic particles and polymer concentrations well below the semidilute-concentrated crossover $c^{* *}$. The latter concentration signifies the point at which the universal structural feature of a semidilute polymer solution, the physical mesh or polymer density-density screening length, becomes comparable to, or smaller than, the monomer size [6]. For $c \ll c^{* *}$, which is the regime relevant to most suspension experiments, a field-theory-inspired and analytically tractable 'thread model' of flexible polymer chains has been previously developed and is employed here $[19,47,48]$.

The second major challenge is of a conceptual nature. It is well known from atomic liquidstate theory that reliable prediction of structure, and especially phase separation, of highly asymmetric (but additive) hard-sphere mixtures is a difficult problem due to its sensitivity to closure approximations [34]. In particular, the classic Percus-Yevick (PY) theory incorrectly predicts the absence of fluid-fluid phase separation [49]. In mixture problems, accurate treatment of the 'cross-correlations' is a very important and demanding task. For the present problem, this involves the packing of polymer segments (not entire coils) against mesoscopic particles. We have found that to properly capture the essential physics within a liquidstate theory framework requires a new approximation scheme (called 'modified PY', m-PY) that accounts for non-local entropic repulsions between polymer segments and the colloid (due to polymer connectivity correlations and conformational perturbations) in a predictive, thermodynamically self-consistent manner.

The goal of this article is not to present the technical details of the m-PY PRISM theory, which are available elsewhere [16,17]. Rather, we first summarize the essential theoretical ideas in section 2, and then concentrate on the structural consequences for which the liquid-state approach makes unique predictions. Prior theoretical results of others for structural questions are limited to the elementary, but still physically complex, problem of one or two particles dissolved in a polymer solution. The polymer segment-colloid-density profiles for a single hard sphere in a dilute or semidilute polymer solution have been exhaustively studied for all $R_{g} / R$ size ratios [27-29]. Field theory, scaling arguments, density functional theory, and selfconsistent mean-field theory have all been utilized to varying degrees and for restricted size 
asymmetry regimes to study the problem of polymer-induced depletion interactions between a pair of particles at infinite dilution $[27,50]$. These results serve as valuable benchmarks for testing the reliability of the PRISM approach in the special limiting situations discussed in section 3. The opposite dilute regime, one polymer in a particle suspension, is also considered in section 3, and is not treatable by scaling or self-consistent mean-field approaches. We then turn to the question of polymer-colloid mixtures with both components present at nonzero concentrations. Our predictions $[16,32]$ for spinodal fluid-fluid phase separation are summarized in section 4 and contrasted with prior theories. In sections 5-7, we present new representative results for all three structural pair correlation functions, on both local and mesoscopic length scales, and in both real and Fourier space. Their physical interpretation is emphasized, and the systematic dependence on controllable system variables is established. The paper concludes in section 8 with a discussion of the present limitations of the theory, and an outlook to the future. Finally, we shall make only brief contact with prior theoretical studies. Detailed comparisons for structural correlations are not possible since the vast majority of prior work is of a statistical thermodynamic nature, and polymer correlations at non-zero concentrations have not been addressed.

\section{PRISM m-PY theory}

We consider the density fluctuations, as quantified by the partial structure factors $\hat{S}_{i j}(q) \sim$ $\left\langle\delta \varrho_{i}^{*}(\boldsymbol{q}) \delta \varrho_{j}(\boldsymbol{q})\right\rangle$ for wavevector $\boldsymbol{q}$, of a binary mixture of polymer chains (composed of $N$ interaction sites or segments) and hard spheres (radius $R$ ) which interact solely via pairdecomposable site-site hard-core repulsions between all species. In obvious matrix notation, the total density fluctuations in Fourier space consist of single-molecule contributions described by a (diagonal) intramolecular structure factor, $\hat{\omega}_{i j}(q)=\hat{\omega}_{j}(q) * \delta_{i j}$, and intermolecular sitesite correlations, $\hat{h}_{i j}(q)$ :

$$
\hat{S}(q)=\hat{\omega}(q)+\varrho^{1 / 2} \hat{h}(q) \varrho^{1 / 2} .
$$

The diagonal matrix of densities, $\varrho_{i j}=\varrho_{j} \delta_{i j}$, defines the number density of colloids and polymer segments. The intermolecular pair correlation functions, $g_{i j}(r)=1+h_{i j}(r)$, describe the relative probabilities of finding a site of species $i$ at a distance $r$ from a site of species $j$ at the origin. The intermolecular hard-core exclusion constraints require $g_{i j}\left(r<\left(\sigma_{i}+\sigma_{j}\right) / 2\right)=0$, where $\sigma_{2}=\sigma_{c}$ is the colloid hard-core diameter, $\phi_{c}=(\pi / 6) \varrho_{c} \sigma_{c}^{3}$ the associated packing fraction, and $\sigma_{1}=\sigma_{p}$ the excluded-volume diameter of a single polymer site (segment); polymer coils, which can interpenetrate, nevertheless cannot intersect their backbones. Note that intramolecular excluded volume enters via the single-polymer-chain structure factor.

The generalized Ornstein-Zernike, or Chandler-Andersen, equation is given by [20,21]

$$
\hat{S}^{-1}(q)=\hat{\omega}^{-1}-\varrho^{1 / 2} \hat{c}(q) \varrho^{1 / 2}
$$

which serves to define the effective potentials, the site-site direct correlation functions, $c_{i j}(r)$. Liquid-state theory approaches are based on the classic idea that the $c_{i j}(r)$ are relatively simple objects that exhibit a spatial range which is of the order of the potential range even if the system builds up collective long-ranged fluctuations. The familiar random-phase approximation (RPA) [6] replaces the $c_{i j}(r)$ with the bare pair potentials, but fails to fulfil the excludedvolume constraints on $g_{i j}(r)$.

Use of these exact formulae is made in PRISM theory by invoking several approximations which we view as physical assumptions that can be tested, either directly by experiments or simulations, or via comparisons of their consequences with more rigorous alternative theories in tractable limiting cases. PRISM theory is fundamentally a theory for structural properties 
deduced not from an approximated free energy, but from approximated equations for the structure factors and pair correlation functions themselves. Besides the well-established use of such liquid-state approaches $[19,21,34]$, and the absence of alternative methods with comparable reaches, our discussion of the m-PY PRISM approach is further motivated by its prediction of a scaling-law limit for large polymers, which is an extension of known field theoretic results for dilute systems. The connection of the corresponding structural correlations to this aspect and to the physical approximations will be one of our central topics. The model and statistical mechanical approximations used in m-PY PRISM theory are the following:

(i) The rigid colloid is described by a single site, leading to $\hat{\omega}_{c}(q)=1$. The structure of a single flexible polymer is assumed to be the same everywhere in the system and to be known a priori (the 'homogeneous pre-averaging assumption' [21,22]):

$$
\hat{\omega}_{p} \equiv \omega(q)=\frac{1}{N} \sum_{\alpha \beta}^{N}\left\langle\mathrm{e}^{\mathrm{i} q \cdot\left(r_{\alpha}-r_{\beta}\right)}\right\rangle \approx \frac{N}{\left(1+q^{2} \xi_{0}^{2}\right)} .
$$

The second approximate equality in equation (3) corresponds to adopting an analytically simplified ideal- (Gaussian-) chain description where the coil radius of gyration $R_{g}=$ $\sqrt{2} \xi_{0}$, or equivalently $R_{g}=\sqrt{2 N} l_{p}$, where $l_{p}$ is proportional to the ideal-walk step, or 'statistical segment' length. Additionally, only site-averaged quantities are considered, and hence specific chain-end effects are ignored [19].

(ii) For the colloidal hard-sphere and athermal polymer components, we adopt the accurate site-site PY approximation: $c_{j j}\left(r>\sigma_{j}\right)=0$, stating that the effective potentials vanish beyond overlap of the sites. This strict implementation of the original Ornstein-Zernike idea has shown its great value for the description of the pure systems [19,21,34], and improvements on it are available.

(iii) For the polymer segment-colloid direct correlation, we have proposed the novel m-PY approximation as a one-parameter extension of the PY closure [16]:

$$
\hat{c}_{c p}(q)=\frac{\hat{c}_{c p}^{s}(q)}{1+q^{2} \lambda^{2}}, \quad \text { with } \quad c_{c p}^{s}\left(r>\frac{\sigma_{c}+\sigma_{p}}{2}\right)=0,
$$

or equivalently in real space:

$$
c_{c p}(\boldsymbol{r})=\int \mathrm{d}^{3} s \frac{1}{4 \pi \lambda^{2}} \frac{1}{|\boldsymbol{r}-\boldsymbol{s}|} \mathrm{e}^{-|\boldsymbol{r}-s| / \lambda} c_{c p}^{s}(s) .
$$

The function $c_{p c}^{s}(r)$ is the analogue of the short-range PY direct correlation function which vanishes for segment-colloid separation beyond contact, and can be interpreted as describing unconnected polymer segments. It must be determined such that the exact core condition for $g_{c p}(r)$ is satisfied. The parameter $\lambda$ introduces the spatial nonlocality of the segment-colloid interactions due to entropic considerations. The spatial convolution form in (5) describes non-local conformational constraints on segmental packing (chain connectivity) within a distance $\lambda$ of the colloidal surface. Such a mediumranged effective interaction becomes necessary in theories employing a pre-averaging assumption for the single-polymer structure since a polymer coil rearranges close to a repulsive surface. Neglect of these rearrangements (equivalent to the standard $\lambda=0$ sitesite PY approximation) within the PRISM theory results in a qualitative overestimate of the polymer segment density close to the particles, thereby entailing a severe underestimation of the induced depletion attraction $[17,51]$.

The m-PY PRISM approach adopts further technical approximations or simplifications when handling specific properties of the system. 
(iv) Polymer correlations at low semidilute concentrations obey universal scaling laws, which are known from scaling arguments and field theoretic calculations [6]. PRISM exhibits an equivalent limit, termed the 'thread' model $[47,48]$, which is analytically tractable and can be considered an approximation to the scaling laws, physically appropriate for dilute and semidilute polymer concentrations, but quantitatively slightly different. The polymer specific scaling laws express the fact that excluded-volume interactions between segments on different coils remain active even if the thickness of the segments is negligible, $\sigma_{p} \rightarrow 0$. Only the polymer segment density relative to the semidilute crossover segmental number density (which defines when polymer coils just begin to interpenetrate and interact), $\varrho_{p}^{*}$, is then relevant for long polymer chains. The dimensionless measure of polymer concentration is $\varrho_{p} / \varrho_{p}^{*}=c / c^{*}=(4 \pi / 3)\left(\varrho_{p} / N\right) R_{g}^{3}$.

(v) Our additional assumption of Gaussian single-chain statistics in (3) corresponds to a mean-field approximation to the polymer correlations with self-avoidance and entails the familiar errors in the scaling-law exponents as thoroughly discussed elsewhere [6]. This approximation is fundamentally different to the often-used literal 'ideal-chain' approximation, which also neglects polymer-polymer interactions. While the former simplifies polymer correlations, the latter totally neglects polymer interactions, treating the coils as an ideal gas. As discussed later, this generally fails for higher polymer densities and/or $R_{g}$.

(vi) The information required to solve the three coupled PRISM integral equations is now specified, given the non-locality parameter, $\lambda$. Significant analytic progress can be made using the Baxter or Wiener-Hopf factorization method $[17,34]$. The parameter $\lambda$ contains much of the many-body physics of depletion interactions and is expected to vary nontrivially with all system parameters, $\phi_{c}, R_{g} / R$, and $c / c^{*}$. In order to achieve a parameterfree theory, a thermodynamic consistency condition is enforced. The excess (non-ideal) chemical potential for inserting a single polymer $\left(\varrho_{p} \rightarrow 0\right)$ into a colloidal suspension of volume fraction $\phi_{c}$ is required to agree when computed from the compressibility and free-energy charging routes. The two formally exact expressions for this quantity are equated yielding a highly non-linear equation for $\lambda[21]$ :

$$
\begin{gathered}
-\left.\int_{0}^{\varrho_{c}} \mathrm{~d} \varrho_{c}^{\prime} N \hat{c}_{c p}\left(q=0, \varrho_{c}^{\prime}\right)\right|_{\varrho_{p}=0}=\left.\frac{\pi \varrho_{c} \sigma_{c} N}{2} \int_{0}^{1} \mathrm{~d} \zeta\left(\sigma_{p}+\zeta \sigma_{c}\right)^{2} g_{c p}^{(\zeta)}\left(\frac{\sigma_{p}+\zeta \sigma_{c}}{2}\right)\right|_{\varrho_{p}=0} \\
+\left.2 \pi \varrho_{c}^{2} \sigma_{c}^{3} N \int_{0}^{1} \mathrm{~d} \zeta \zeta^{2} \frac{\partial g_{c c}^{(\zeta)}\left(\zeta \sigma_{c}\right)}{\partial \varrho_{p}}\right|_{\varrho_{p}=0} .
\end{gathered}
$$

The compressibility route emphasizes long-wavelength information encoded in the segment-colloid direct correlation function via charging up (thermodynamic path integration) of the colloid density. On the other hand, the free-energy route emphasizes the local contact value of the real-space segment-colloid pair correlation function via a growth process whence the diameter of the colloidal spheres is increased from zero to its full value. Requiring $\lambda$ to be chosen such that these two very different exact routes yield identical polymer-insertion chemical potentials is a powerful consistency constraint on the structural correlation functions. Exact numerical determination of $\lambda$ from the above equations is difficult because thermodynamic integrations are required. Thus, on the basis of exact analytic analysis of several limiting cases, we proposed an approximate interpolation formula which is quite accurate (satisfies thermodynamic consistency to within $15 \%$ or less for all parameter values) $[16,17]$ :

$$
\lambda^{-1}=\xi_{0}^{-1}+\frac{1+2 \phi_{c}}{1-\phi_{c}} \frac{\lambda_{1}}{\sigma_{c}},
$$


where $\lambda_{1}=1+\sqrt{5}$. The limit $\phi_{c} \rightarrow 0$ corresponds to the (still many-body) problem of one polymer and one colloid. In nearly quantitative agreement with mean-field theoretic studies [27-29,50], and physical intuition, the non-locality length scale is then predicted to reduce to the polymer correlation length $\left(\xi_{0}=R_{g} / \sqrt{2}\right)$ if $R \gg R_{g}$, and a fraction of the colloid radius, $2 R / \lambda_{1}$, if $R_{g} \gg R$. For $R_{g} \ll R, \lambda$ is nearly independent of colloid concentration since the coils can fit into the voids between particles and screening of the depletion layer around one colloid by other particles is not effective. Such a situation is implicit in using one-component colloid models with effective ( $\phi_{c}$-independent $)$ depletion attraction pair potentials between particles. However, for $R_{g} \gg R$, long polymers can wrap around the colloids, and $\lambda$ is predicted to monotonically decrease with increasing colloid volume fraction. This reflects many-particle screening of the repulsive polymercolloid interactions by colloidal density fluctuations, which reduces entropic attraction resulting in a filling in of the depletion layer surrounding the colloidal particles.

(vii) To determine $\lambda$ beyond vanishingly small polymer concentrations, we have employed the 'blob scaling' concept well known in polymer physics [6]. The idea is that when $c>c^{*}$ in semidilute solution the physical mesh, or equivalently polymer densitydensity correlation or screening length, $\xi$, is the relevant length scale, not $R_{g}$. Note that $\xi=R_{g} \hat{\xi}\left(R_{g} / R, c / c^{*}, \phi_{c}\right)$ is explicitly calculated within our theory and not obtained from blob scaling. In the absence of particles, thread PRISM theory predicts [19]

$$
\frac{1}{\xi}=\frac{1}{\xi_{0}}+4 \pi \rho_{p} l_{p}^{2}=\frac{1+(3 / \sqrt{8}) c / c^{*}}{\xi_{0}} .
$$

If one employs the known scaling law for the effective statistical segment length in semidilute athermal solutions, $l_{p} \sim \rho_{p}^{-1 / 8}$, then (8) agrees with experiment, field theory, and scaling arguments for athermal polymer solutions $[6,19]$. In the limit of $\phi_{c} \rightarrow 0$, at non-zero polymer concentrations, $\xi_{0}=R_{g} / \sqrt{2}$ in (7) is replaced by the mesh length, i.e. $\xi_{0} \rightarrow \xi$. For $R \gg R_{g}$, the depletion layer and non-locality parameter are predicted to be given by $\xi$, in agreement with field theory and scaling arguments for a polymer solution near a large colloid or effectively a planar surface $[28,50]$. At non-zero colloid concentration, the effective polymer correlation length, $\xi$, will depend on $\phi_{c}$. A naive idea would be that since the colloids occupy a finite fraction, $\phi_{c}$, of the available space, the semidilute crossover concentration, $c^{*}$, would decrease by the factor of $\left(1-\phi_{c}\right)$ in equation (8). Numerical studies $[16,17]$ of the full $\mathrm{m}$-PY equations reveal that this idea is very accurate in the colloid regime of $R_{g} \ll R$. However, in the opposite extreme nanoparticle regime, this naive idea does not hold ${ }^{4}$, and equation (8) remains quite accurate, implying an insensitivity of $\xi$ to the colloid volume fraction for very long polymers.

We now turn to the systematic examination of structural correlations. Common system parameter choices are made to allow cross-comparisons between the three distinct correlation functions and partial scattering structure factors. We shall present results for three values of $R_{g} / R=0.1$ (representative of common colloid systems), 1.0 (the crossover case), and 5 (easily achievable with surfactant micelles, dendrimers, or proteins as the 'nanoparticle'). Results for a 'moderate' and 'high' value of the colloid packing fraction are discussed, $\phi_{c}=0.2$ and 0.45 , respectively. Analytic results in the asymptotic $R_{g} / R \rightarrow \infty$ and $R_{g} / R \rightarrow 0$ limits are also presented.

Finally, we point out that comparison of experimental scattering patterns with our predictions is direct, and no convolutions with a single-polymer form factor are necessary

${ }^{4}$ We find $\xi_{0} / \xi=1+(3 / \sqrt{8})\left(c / c^{*}\right)\left(1-0.42 \phi_{c}\right) /\left[\left(1-\phi_{c}\right)\left(1+2 \phi_{c}\right)\right]$ for $R_{g} \gg R$, which gives an initial increase of $c^{*}\left(\phi_{c}\right)$ by $23 \%$. 


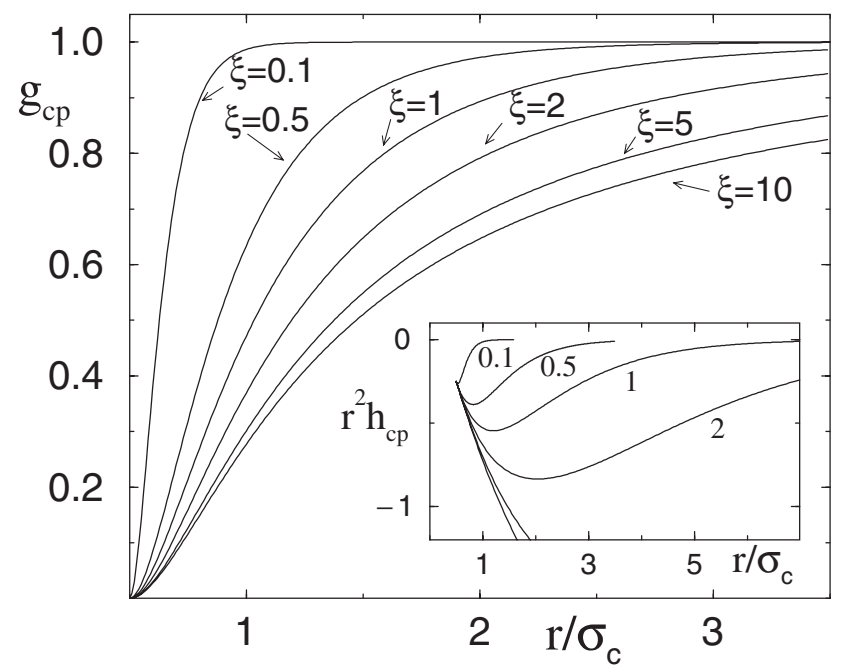

Figure 2. The polymer-colloid pair correlation function, $g_{c p}(r)$, in the dilute and semidilute polymer concentration region exhibiting the polymer segment depletion layer close to an isolated colloidal sphere for various polymer correlation lengths $\xi$ (in units of $\sigma_{c}$ ) as labelled. The inset shows the same data but as $r^{2} h_{c p}(r)=r^{2}\left(g_{c p}(r)-1\right)$ which reveals the long-ranged tail of the depletion layer for large $R_{g} / R$.

since we model polymers at the elementary segmental scale; for a discussion of an alternative approach via polymer-centre-of-mass correlations, see [52]. Here, for example, the total scattering function is given by the standard formula:

$$
I(q)=\varrho_{c} b_{c}^{2} P(q) S_{c c}(q)+\varrho_{p} b_{p}^{2} S_{p p}(q)+2 \sqrt{\varrho_{c} \varrho_{p}} \sqrt{P(q)} b_{c} b_{p} S_{c p}(q),
$$

where $b_{c}$ and $b_{p}$ are the colloidal and polymer segment scattering lengths, respectively, and $P(q)$ is the particle form factor. The traditional emphasis is on extracting the colloidal partial structure factor, $S_{c c}(q)$. We hope our calculations will motivate future neutron scattering experiments to directly measure the polymer scattering functions.

\section{Dilute limits}

The dilute limits are relatively well understood and provide important insight into the structural modifications in finite-composition mixtures. We focus on the structural aspects, and refer the reader elsewhere [17] for the thermodynamic results.

\subsection{Dilute particles}

In this limit the polymer correlations are unaffected by the presence of particles. The polymer solution is characterized by a density-density correlation (or screening) length, $\xi$, also known as the physical mesh length or blob size. In dilute polymer solutions, $\xi$ reduces to $R_{g} / \sqrt{3}$ $\left(R_{g} / \sqrt{2}\right.$ within our simple approximation of (3)). The polymer segment density at a distance $r$ from a particle is given by $\varrho_{p} g_{c p}(r)$. Representative results for the polymer segment-colloid radial distribution function are given in figure 2. All display the classic 'depletion hole' suppression below the random value of unity and a monotonic approach to unity as $r \rightarrow \infty$. There are three important aspects of these results. 
(a) Close to contact, the pair correlations grow in a power-law fashion, $g_{c p}(r) \sim$ $([r-R] / \lambda)^{1 / v}$ (with Flory exponent $v=\frac{1}{2}$ in our mean-field-like description), as required from the wall virial theorem [27-29,50]. It connects the osmotic pressure of the polymer solution ( $\Pi \sim k T / \xi^{3}$ for semidilute concentrations) to the force per area that the segments exert. The force is simply proportional to segment density, which is $\varrho_{p} g_{c p}\left(R+\sigma_{p}\right) \sim$ $\varrho_{p}\left(\sigma_{p} / \xi\right)^{x}$. Equating the pressures determines $x=1 / v[28,50]$. Gratifyingly, the various theoretical estimates of the (universal) coefficients of the quadratic growth law agree to within $\sim 20 \%$ error in both the $R \gg R_{g}$ extreme colloid and $R_{g} \gg R$ extreme nanoparticle limits [17].

(b) In the extreme colloid regime, the particle appears to the polymer as a flat wall. The width of the depletion layer is the polymer correlation length, $\xi$.

(c) In the extreme nanoparticle limit, the polymer-particle-density profile changes qualitatively. Two length scales emerge: a local length of order the particle radius due to the direct perturbation of (local) polymer conformational entropy by the particle, and a longer length scale of order $\xi$. For intermediate separations, the profile increases in $\mathrm{a} \sim r^{1 / \nu-d}$ power-law manner reflecting the self-similar chain connectivity correlations on length scales smaller than the mesh size. The inset in figure 2 highlights the new longer-length-scale feature which clearly emerges when $\xi \gg R$. Whereas the existence of a narrow (of order $R$ ) component of the depletion layer for $R_{g} \gg R$ is rather obvious, the aspect that a long polymer (considering the dilute case for simplicity) cannot totally balance the disturbance of a point repulsion on distances shorter than $R_{g}$ warrants further discussion, especially since its consequences appear to have been overlooked in several prior works [53-55]. It is equivalent to the statement that order$N$ segments of the chain rearrange when a small particle is added. The free-energy increase on adding a few particles is proportional to the number of segments independently displaced by each of the particles. It can be estimated from the average number of polymer segments within the sphere volume, which needs to be corrected by the number of correlated segments belonging to the same polymer strand. Thus it scales like $\delta F / V k T \sim \varrho_{p} \varrho_{c} \sigma_{c}^{d-1 / v} l_{p}^{1 / v} \sim c_{p} \varrho_{c}\left(N \sigma_{c}^{d-1 / v} l_{p}^{1 / v}\right)$ where $l_{p}$ is the Kuhn-segment length, $d$ the dimension of space, and the latter number density $c[29,54,56]$. Therefore, on a per-coil basis the free-energy increase is of order $N$, which requires the whole chain to rearrange around the particle. The second virial cross-coefficient in this limit, $B_{2}^{c p}=\partial^{2}(\delta F / V k T) /\left(\partial c_{p} \partial \varrho_{c}\right)=-\frac{1}{2} \hat{h}_{c p}(q=0) \sim N \sigma_{c}^{d-1 / v} l_{p}^{1 / v}$, thus grows with $N$, and as it is given by the integral of the correlated part of the segment profile, $g_{p c}(r)$ can approach unity only for $r \sim R_{g}$.

The extreme nanoparticle regime results were previously known only from the field theoretic work of Eisenriegler and co-workers [27-29]. Moreover, their experimental significance was only very recently realized from the studies by Kulkarni et al [57] of the second virial coefficient of small proteins dissolved in polymer solutions. These experiments discovered a novel non-monotonic dependence of the consequences of depletion attractions on polymer concentration which originates in the different concentration dependences of the two depletion layer lengths. Sensitivity to solvent quality, and hence polymer-polymer correlation length, was also discovered. The experimental observations have been shown to be well described by PRISM theory [57].

It is significant that alternative polymer-based treatments of the nanoparticle regime have come to conflicting, and apparently incorrect, conclusions. The early analysis of de Gennes [54] suggests that the depletion attraction effect is negligible, and all mixtures are miscible, if $R<\xi$, thereby seemingly missing the long-range aspect discussed above. Recent works 
by Odijk [55] and Tuinier et al [53] come to similar conclusions, that the range of depletion attractions between two spheres is controlled solely by particle size and is $\sim 3 R$. These workers employ an uncontrolled 'superposition of one-particle depletion layer approximation', which is not invoked in the field theoretic or integral equation approaches. The particle-particle second virial coefficient for ideal coils is reported to remain positive under dilute-polymer $\left(c<c^{*}\right)$ conditions [53], in disagreement with the field theoretic [27] and PRISM [17, 57] predictions, and the experimental observation [11,13,15,32] of fluid-fluid demixing at $c<c^{*}$ when $R_{g}>R$.

The classic depletion effect is generally discussed at the level of an effective entropic attraction, $V(r)$, between a pair of particles mediated by the polymer solution. If $c \ll c^{*}$, it is the theoretical foundation for effective one-component and related statistical thermodynamic approaches. Within the PY closure for particle-particle direct correlations, m-PY PRISM theory yields, in units of $k T$, a result showing clearly the connection to the polymer correlations:

$V(r)=-\ln (1+W(r)), \quad W(r)=\Theta(r-2 R) \int \frac{\mathrm{d}^{3} q}{(2 \pi)^{3}} \mathrm{e}^{-\mathrm{i} q \cdot r} \varrho_{p} c_{c p}^{2}(q) S_{p p}(q)$.

$W(r)=g_{c c}(r)-1$ is analytically accessible but sufficiently cumbersome that we refrain from presenting it here. Alternatively, if one adopts the hypernetted chain (HNC) closure for colloid-colloid direct correlations, one obtains $V(r)=-W(r)[17,51]$. In the $c / c^{*} \rightarrow 0$ ultradilute-polymer limit, the HNC- and PY-based expressions agree. Hence, we analyse this regime to make contact with the classic AO model. The latter is formulated in the $R \gg R_{g}$ regime, for which one obtains

$$
V_{\mathrm{AO}}=\frac{3}{2} \frac{c}{c^{*}} \frac{R}{R_{g}}[1-H]^{2},
$$

where $H=(r-2 R) / 2 R_{g}$ is the surface-to-surface separation of the large colloids, and the spatial range of $2 R_{g}$ follows directly from the modelling of a polymer coil by a hard sphere of radius $R_{g}$. On the basis of self-consistent mean-field results [50], it is sometimes suggested that the width of the depletion layer within an AO framework should be $\sqrt{4 / \pi} R_{g}$. This small quantitative modification increases $V_{\mathrm{AO}}(2 R)$ by a factor of 1.27 , and increases the spatial range of the depletion potential by $12 \%$. The m-PY PRISM result in the analogous limit is [17]

$$
V_{\mathrm{m}-\mathrm{PY}}=\frac{27}{8} \frac{c}{c^{*}} \frac{R}{R_{g}}\left[1+(5 / 9) X+(X / 3)^{2}\right] \mathrm{e}^{-X},
$$

where $X=(r-2 R) / \xi_{0}$. The PRISM and AO depletion potentials are qualitatively identical in the $R \gg R_{g}$ ultradilute-polymer limit. Quantitatively, the amplitude of the PRISM result is roughly a factor of 2 larger than the AO one, and its range is modestly longer (see figure 3). Both these differences are connected to the fact that PRISM theory treats the polymer as a fluctuating coil and (approximately) accounts for the loss of both translational and conformational (orientational) entropy when confined between two large colloids. Field theory also includes the latter physical effect which results in a non-zero $V(H)$ beyond $H=1$ and a contact strength which is larger than the AO result [28]: $V(H=0)=3\left(c / c^{*}\right)\left(R / R_{g}\right) \ln 2$.

Figure 3 presents results for the normalized potential of mean force, $-k T \ln g_{c c}(r)$, of m-PY PRISM theory at several reduced polymer concentrations and two $R_{g} / R$ values. The $R_{g} / R=0.1$ case is quite close to the extreme colloid limit discussed above. Interestingly, the spatial range of the PRISM depletion potentials is a non-monotonic function of reduced polymer concentration, reflecting a subtle and non-trivial dependence of the amplitude at contact and polymer correlation length on $c / c^{*}$. Only for larger polymers, (e.g., $R_{g}=R$ in figure 3 ) does the expected trend emerge that the potential range decreases as $\xi$, the blob size. 


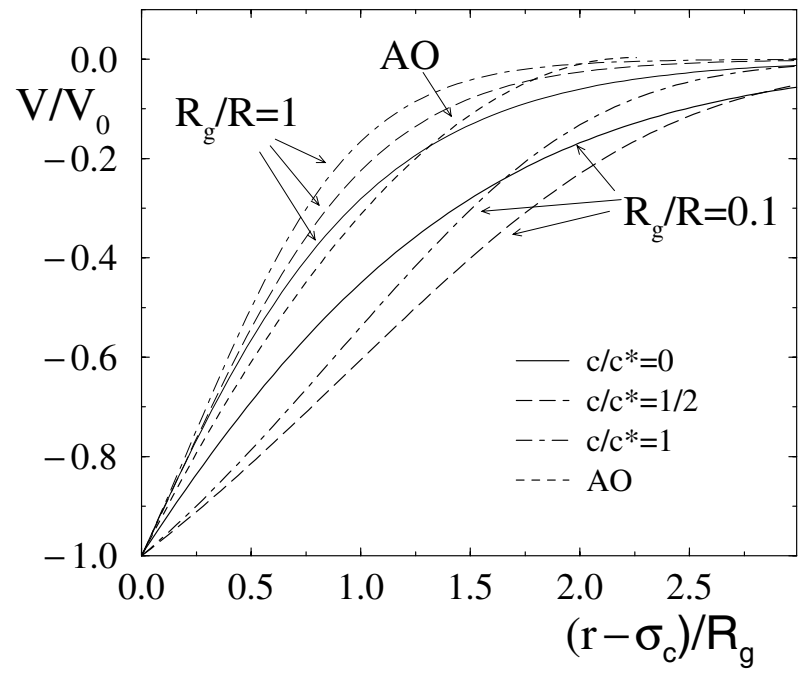

Figure 3. Normalized polymer-induced potential of mean force, $V(r) / V_{0}=-\log \left(g_{c c}(r)\right) / V_{0}$, where $V_{0}=\log g_{c c}\left(\sigma_{c}\right)$, versus reduced distance, $\left(r-\sigma_{c}\right) / R_{g}$, in the limit of vanishing colloid concentration and for the labelled polymer concentrations. Thick curves correspond to $R_{g} / R=0.1$ and thin curves to $R_{g} / R=1$. The short-dashed curve shows the Asakura-Oosawa result with a depletion layer of $(2 / \sqrt{\pi}) R_{g}$.

From a broad theoretical perspective, we believe the qualitative agreement between the field theoretic and m-PY PRISM approaches (where so far it has only been possible to analytically handle mean-field exponents) for the polymer-particle-density profiles (and thermodynamics $[16,17]$ ) over the entire range of $R_{g} / R$ provides a solid foundation for extension of the integral equation theory to the non-zero- $c / c^{*}$ and non-zero- $\phi_{c}$ mixture regimes of primary experimental interest.

\subsection{Dilute polymers}

The opposite problem of one or two polymers in a hard-sphere fluid is far less understood [6,26]. The central statistical geometry problem is how polymer coils can 'fit into' the free volume of a colloidal fluid, which in the dilute-polymer limit is not perturbed by their presence. When $R_{g}>R$, the popular approximation of an open, fractal polymer coil as a solid particle is clearly invalid.

Examples of the polymer-colloid profiles are given in figure 4. As expected, at low colloid volume fraction the behaviour is similar to that shown in figure 2. For large polymers, the depletion hole has a local and long-range component. As the colloids are densified, the local depletion layer narrows due to many-particle screening of the direct perturbation of the polymers by the repulsive polymer-colloid interactions. The dependence of the depletion layer on colloid density renders simple effective potential approaches inapplicable in general. In the limit of vanishingly small polymers, $R_{g} \ll R$, the polymer correlations can be found explicitly. The segment density jumps from 0 to $\varrho_{p} /\left(1-\phi_{c}\right)$ at contact. Thus for $R_{g} / R \rightarrow 0$ the polymer correlations directly follow the colloidal ones:

$$
\hat{h}_{c p}(q)=\frac{-1}{1-\phi_{c}} \hat{F}(q) \hat{S}^{\mathrm{HS}}(q) \quad \text { where } \hat{F}(q)=\int \mathrm{d}^{3} r \mathrm{e}^{\mathrm{i} q \cdot r} \Theta(R-r) .
$$

$\hat{F}(q)$ is the scattering amplitude of the empty polymer regions whose centres are correlated 


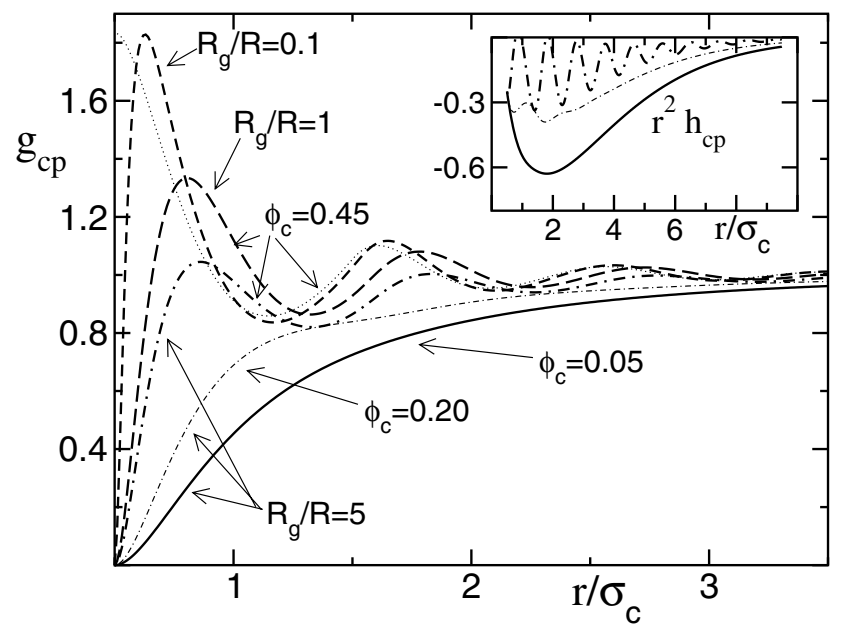

Figure 4. Polymer-colloid pair correlation functions, $g_{c p}(r)$, for dilute polymers $\left(\varrho_{p}=0\right)$ and colloid packing fraction and size ratio as labelled. The (unlabelled) thin dotted curves give the asymptotic result for $R_{g} / R \rightarrow 0$ corresponding to equation (13). The inset shows the long-ranged part of the depletion layer for the size ratio $R_{g} / R=5$ (line style as in the main panel) which is apparent in a plot of $r^{2} h_{c p}(r)=r^{2}\left(g_{c p}(r)-1\right)$.

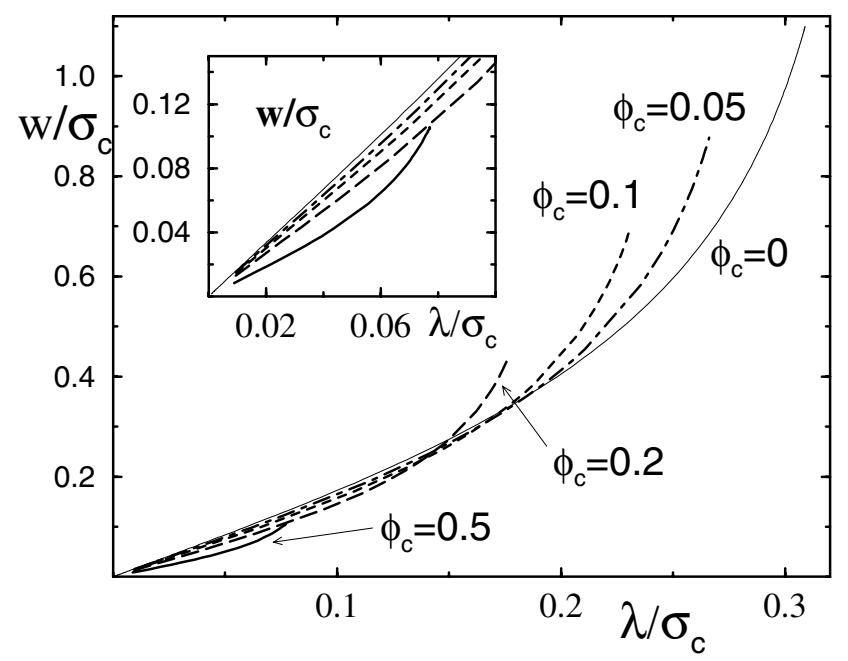

Figure 5. Local width of the polymer segment depletion layer defined by $g_{c p}\left(\sigma_{c} / 2+w\right)=\frac{1}{2}$ versus the colloid-polymer interaction length $\lambda$ for the labelled colloid concentrations and at vanishing polymer concentration. Along the curves, the polymer size varies in the range $0.03 \leqslant R_{g} / R \leqslant 280$, and $\lambda$ is given by equation (7). The result for vanishing colloid concentration, the thin solid curve, also describes the width at finite polymer concentration, where the polymer correlation length is given by equation (8). The inset presents an enlarged view relevant for small polymer correlation lengths.

according to the hard-sphere structure factor $\hat{S}^{\mathrm{HS}}(q)$ of the particles. The particle form factor is given by $P(q)=\left|\hat{F}(q) /\left(4 \pi R^{3} / 3\right)\right|^{2}$, and also appears in the dilute-polymer correlations, $\hat{S}_{p p}(q)=(4 \pi / 3) R^{3} \varrho_{p}\left[\phi_{c} /\left(1-\phi_{c}\right)^{2}\right] P(q) \hat{S}^{\mathrm{HS}}(q)$ for $R_{g} \ll R$. Hence, at high colloid volume fraction there appears an oscillatory packing of the polymers on the colloid-size length 
scale. This represents an 'imprinting' of the colloidal structure and free-volume correlations on the polymer segment spatial organization. This effect was first observed by Louis et al [42], considering the polymers as phantom spheres. As figure 4 shows, where the limit corresponding to (13) is included, it is present for all $R_{g} / R$ ratios even in the nanoparticle regime where the polymers must 'go around' many particles in the fluid. However, careful examination of $g_{c p}(r)$ curves for $R_{g} \gg R$ (see the inset) reveals that the long-range component of the depletion hole is not destroyed, but just becomes of smaller relative amplitude and more difficult to resolve.

A summary of our attempt to quantify the local width of the depletion layer is given in figure 5. The partial collapse of the results over a four-orders-of-magnitude variation of size asymmetry suggests a strong connection between the layer width, $w$, and the fundamental nonlocality parameter, $\lambda$. The relatively modest variation of the width is remarkable. At higher colloid volume fractions the layer width becomes a unique function of $\lambda$, which only mildly splays out as $\phi_{c}$ is decreased. Both $w$ and $\lambda$ saturate at finite values in the limit $R_{g} / R \rightarrow \infty$ since the relevant length scale is then the colloid size. Polymer-polymer correlations in the dilute limit are discussed in section 7.

\section{Fluid-fluid phase separation}

The fluid-fluid spinodal instability lines, and corresponding critical points, predicted by m-PY PRISM theory are shown in figure 6 . The novel trend is that the homogeneous mixed phase is stabilized as the ratio $R_{g} / R$ increases. As discussed in great depth elsewhere [16,32], the predicted trend is in agreement with recent experiments on model athermal polymer-colloid suspensions when fluid-fluid phase separation is the thermodynamically stable transition $\left(R_{g} / R>0.3\right)$. However, all the classic theories that we are aware of $[23,24,30]$, and the recent density functional approach [59], predict the opposite shift of fluid-fluid phase boundaries with size asymmetry. The use of the PRISM dilute-polymer-insertion chemical potential in the classic theories does not correct this qualitative error [32]. Thus, the essential importance of non-zero polymer concentration, for which there are direct and (colloid-) induced polymer-polymer interactions, has been unequivocally established [32].

In the extreme colloid limit, $R \gg R_{g}$, the spinodal curves approach a limiting form. This might be interpreted as suggesting that an effective one-component, simple-liquid-type approach is adequate for small polymers, perhaps of the very short-range adhesive-hard-sphere (AHS) type [60]. However, the critical point of the AHS model is located at $\phi_{c} \approx 0.1$, versus the prediction of two-component PRISM theory of a very concentrated value. Indeed, PRISM theory predicts that the critical point shifts to higher colloid concentrations as $R / R_{g}$ increases, in qualitative agreement with the phantom-sphere free-volume model [30], the behaviour of highly asymmetric hard-sphere mixtures [25], and one-component attractive-square-well or Yukawa-fluid models [60,61].

Another generic feature of figure 6 is the absence of spinodal phase separation at a sufficiently small colloid volume fraction of $\phi_{c}=1 / 22 \approx 0.045$. The origin of this prediction is that with decreasing $\phi_{c}$ the value of the reduced polymer concentration required for phase separation exceeds $c^{*}$. Since we have considered athermal, good-solvent conditions for the polymers, when $c>c^{*}$ repulsive interchain interactions systematically reduce the spatial range of correlated polymer density fluctuations [6], and hence depletion effects, resulting in the predicted behaviour. For $R \gg R_{g}$ and low $\phi_{c}$, the depletion potential between two particles is generally large compared with the thermal energy. Hence, the issue of the closure approximation for the colloid-colloid direct correlations (HNC versus PY) becomes relevant [17]. Such a concern also applies to all prior classic free-energy approaches [23,24,30], including recent density functional theories [59]. 


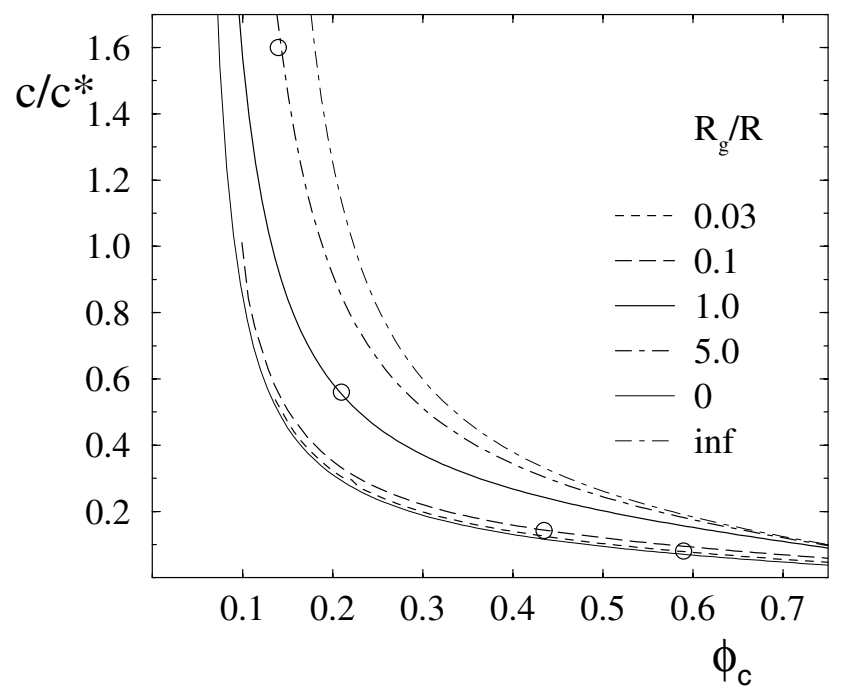

Figure 6. Spinodal curves for various size ratios. The critical points are marked by circles, and thin lines indicate the limits for small (thin solid curves) and large (thin dot-dashed curves) polymer-to-colloid size ratios $R_{g} / R \rightarrow 0(\infty)$, respectively.

The spinodal predictions of PRISM theory for the extreme nanoparticle regime, $R_{g} \gg R$, also contain several novel aspects. The qualitative failure of the AO model is not surprising [24], and alternative polymer-based approaches are relatively few. The de Gennes and Tuinier et al $[53,54]$ approaches discussed in section 3.1 ignore the long-range component of the polymer-mediated attraction. This results in a massive weakening of the depletion effect in the nanoparticle regime, and suggests that no phase separation occurs if $R \ll \xi$ and $c<c^{*}$. However, this disagrees with figure 6 (and experiments [11,13,14,32]) which predicts that phase separation is possible when $R \ll \xi$ and $c<c^{*}$.

Sear has recently presented a simple theory in the phantom-sphere free-volume model spirit for the $R \ll R_{g}$ case [62]. Polymer coils were taken to be non-interacting and ideal, even well into the semidilute regime [6] where demixing is predicted with a $\phi_{c}$ at the critical point that decreases rapidly to zero proportional to the ratio $\left(R / R_{g}\right)^{2}$ due to 'many-body effects'. This latter prediction contradicts figure 6 where the critical colloid volume fraction deep in the semidilute regime approaches a limiting value of 0.12 , typical of a van der Waals-type fluid experiencing weak, long-range attractions. We believe that the qualitative discrepancy is due to the Sear theory not taking into account direct polymer-polymer repulsions and physical mesh formation for $c>c^{*}$, and colloid-induced polymer-polymer attractions at finite polymer concentrations. The direct polymer repulsions are most important in athermal good solvents and when $R_{g} \gg R$, but are also present even for so-called $\Theta$ solvent conditions where ideal polymer solution behaviour fails dramatically above $c^{*}[6]$.

Finally, in the extreme nanoparticle limit the spinodal curves in figure 6 also approach a limiting value. This behaviour arises from the prediction of m-PY PRISM theory that the colloids induce an attractive second virial coefficient between dilute polymers which scales with coil volume [17]. Physically, the extreme nanoparticle limit is akin to the classic polymersolvent problem [6], where the critical point is well known to be correlated with $c^{*}$. For our present problem, the 'solvent' is a mesoscopic particle and the effective colloid-induced attraction between polymer segments is now $\phi_{c}$-dependent. Since the extreme nanoparticle 
regime is characterized by long-range, but weak, depletion effects we believe that our prediction is very reliable.

We now return to our major focus, structural correlations at finite compositions of both components. Polymer concentration will generally be reported in dimensionless units reduced by the value at the spinodal, $c_{s}$. Figure 6 provides the information required to convert this to the classic reduced variable, $c / c^{*}$. Results for a value of $c / c_{s}$ close to unity will be emphasized in order to establish the maximum realizable structural changes in the homogeneous phase.

\section{Colloid-colloid correlations}

An example of the influence of polymers on colloid real-space packing is given in figure 7 for several values of the size asymmetry. The case shown is that of a high colloid volume fraction, but the basic trends are the same at lower values of $\phi_{c}$. The primary effect is the expected enhanced local clustering of hard spheres due to the polymer-mediated depletion attraction. This phenomenon is enhanced (decreased) in amplitude (spatial range) as $R_{g} / R$ decreases, consistent with classic ideas about the role of attractions in fluid structure due to van der Waals and their modern formulation by Weeks, Chandler and Andersen (WCA) [63]. For the smallest-polymer case corresponding to a very short-range attraction, the form of $g_{c c}(r)$ begins to resemble the classic AHS model result [64], including the feature of a highly distorted second solvation shell. In the large-polymer limit, one can show that the deviation between $g_{c c}(r)$ and its pure-hard-sphere analogue is proportional to $R / R_{g} \ll 1$. This makes visualization of structural changes in the nanoparticle regime difficult, but the long-range depletion mechanism discussed in section 3 is still present and observable in wavevector space (see below). Explicitly, we find for $R_{g} / R \rightarrow \infty$

$g_{c c}(r) \rightarrow g^{\mathrm{HS}}\left(r / \sigma_{c}\right)+b \frac{R}{r} A\left(\phi_{c}\right)^{2} \mathrm{e}^{-r / \xi_{c}}, \quad b=4 \pi R l_{p}^{2} \rho_{p}=\frac{3}{2} \frac{c}{c^{*}} \frac{R}{R_{g}}$

where $g^{\mathrm{HS}}$ is the PY hard-sphere pair correlation function, and the (positive) Lorentzian term describes the long-ranged attraction-induced structure. It gets suppressed with increasing $R_{g}$ and colloid packing fraction since $A\left(\phi_{c}\right)=\left(1+2 / \lambda_{1}\right)\left(1-\phi_{c}\right) /\left(1+2 \phi_{c}\right)$. Only the leading terms on small or large scales, $r / \sigma_{c}$ or $r / \xi_{0}$, respectively, are given. The collective correlation length, $\xi_{c}$, increases from the polymer correlation length to infinity when approaching the mean-field spinodals:

$\frac{\xi_{0}}{\xi_{c}}=1-\frac{3}{\sqrt{8}} \frac{c}{c^{*}}\left(f\left(\phi_{c}\right)-1\right) \quad$ where $f\left(\phi_{c}\right)=\frac{\phi_{c}\left(6 \lambda_{1}+1-4 \phi_{c}\right)}{2\left(1-\phi_{c}\right)\left(1+2 \phi_{c}\right)}$.

The quantity $f\left(\phi_{c}\right)$ is the segment-segment contact value for segments from two different coils, which increases from its value 0 , without particles, to large values with increasing $\phi_{c}$; see figure 15 in section 7 . These $R_{g} \gg R$ structural results again suggest that the polymerinduced depletion can be considered in a van der Waals-like perturbative spirit, and that its amplitude is small and given by the locally replaced polymer segment density, $\varrho_{p} l_{p}^{2} R$. This parameter also determined the chemical potential for inserting a single sphere into a polymer fluid as discussed in section 3.1. The intuitive picture, supported by m-PY theory (and field theory results for $\phi_{c}=0$ ), is that this dimensionless interaction parameter depends linearly on polymer concentration throughout the dilute- and semidilute-concentration region since it arises from the interaction of the particle with an isolated polymer strand. It gets renormalized only when the polymer mesh size becomes comparable to the particle size, $\xi \cong R$. Hence, we are rather confident that (14) describes the colloid structure qualitatively up to values of $c / c^{*}$ such that $\xi \cong R$. 


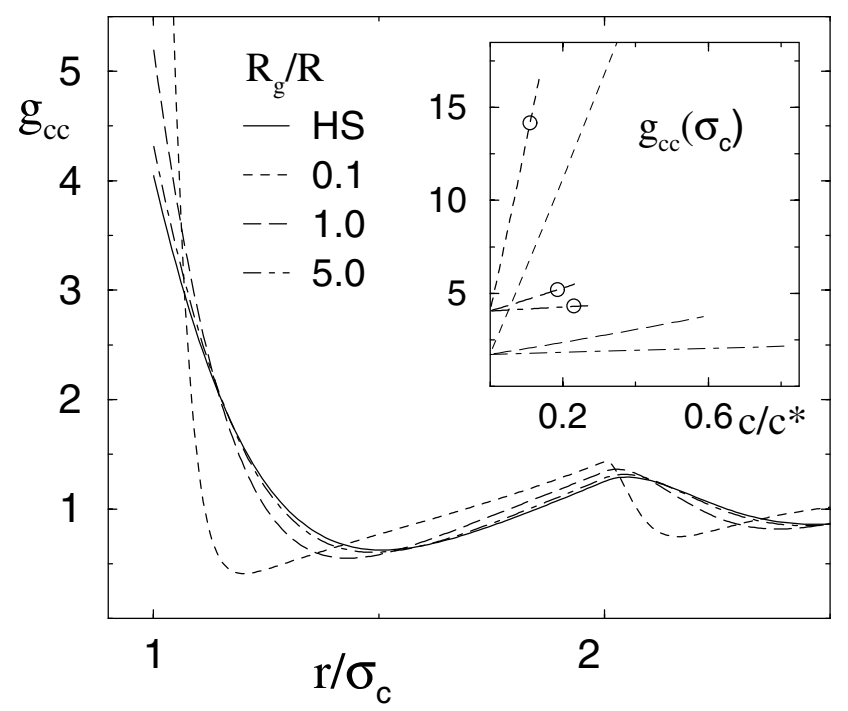

Figure 7. Colloid pair correlation functions, $g_{c c}(r)$, at colloid packing fraction $\phi_{c}=0.45$ and the polymer concentration at a fixed relative distance to the spinodal, $c / c_{s}=0.8$, for the size ratios as labelled; the solid curve (HS) gives the result for hard spheres. The thick curves in the inset give the contact values for the three polymer sizes as a function of the polymer concentration; circles mark the ones of the main figure. Thin curves indicate the corresponding contact values at $\phi_{c}=0.20$.

The enhancement of the contact value of $g_{c c}(r)$ increases monotonically and essentially linearly with polymer concentration, as seen in the inset of figure 7 for two colloid volume fraction cases. It is interesting to observe that the enhancements relative to the pure hardsphere value are larger for the lower- $\phi_{c}$ case. This is a consequence of the increased ability of short-range attractions to induce local reorganization when the fluid is more compressible. The linear dependence of the contact value on polymer concentration is presumably related to our use of the PY closure for the colloid-colloid direct correlations, and hence may be less reliable when $R \gg R_{g}$ in analogy with prior studies of hard-sphere mixtures [49,58].

The colloidal structure factor displays the classic wide-angle peak at $q_{p} \approx 7 \sigma_{c}^{-1}$ which is a quantitative measure of the degree of coherent order of the local 'cage'. A zero-adjustableparameter comparison of PRISM theory with recent light scattering measurements [7] on model athermal polymer-colloid suspensions under near-triple-point conditions is given in figure 8. Good agreement is found at both small and large wavevectors. Earlier analysis of one-component pair potential theories required fits with strongly underestimated polymer concentrations, as pointed out in [42], where a polymer-phantom-sphere approach achieved descriptions of comparable quality to figure 8 upon adjusting $c / c^{*}$ somewhat. The deviations around the wide-angle peak for the smallest-polymer sample require further investigation due to possible experimental difficulties. The inset shows the corresponding predictions for the colloidal radial distribution function. Interestingly, despite the fact that the three samples are all at different values of $\phi_{c}, c / c^{*}$, and $R_{g} / R$, the $g_{c c}(r)$ curves nearly collapse onto a single curve. This may be a consequence of the experiments being performed near the triple point of each system. Future video microscopy experiments could perhaps test this prediction.

As phase separation is approached, very large enhancements of the long-wavelength fluctuations emerge, which can be quantified by the dimensionless colloidal osmotic compressibility, $S_{c c}(0)$. The agreement between theory and experiment in figure 8 at small 


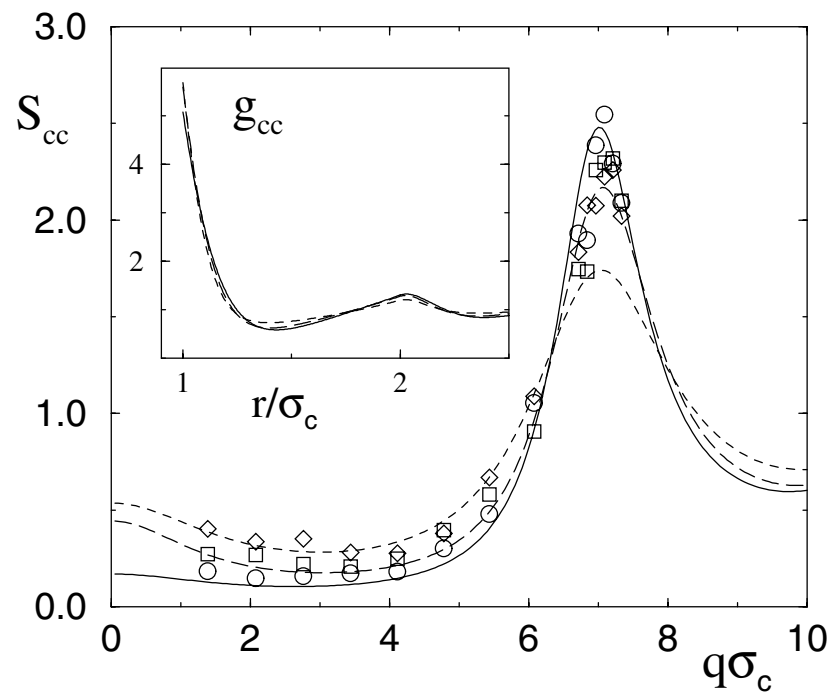

Figure 8. Dimensionless colloid structure factors compared to the experimental data of Moussaid et al [7]. The parameters are $\left(\phi_{c}, c / c^{*}, R_{g} / R\right)=(0.333,0.13,0.24$; short dashes and $\diamond),(0.404$, $0.13,0.37$; long dashes and $\square)$, and $(0.444,0.10,0.57$; solid curve and $\bigcirc)$. The inset shows the corresponding colloid pair correlation functions $g_{c c}(r)$ with the same line styles.

wavevectors is very significant, since $S_{c c}(0)$ is nearly an order of magnitude larger in the polymer-colloid suspensions than the analogous pure-hard-sphere fluid [7]. Very recently, quantitative comparisons of osmotic compressibility data obtained using the turbidity technique have been obtained by Ramakrishnan et al [33] for a large set of model athermal polymercolloid suspensions. Significant errors of both a quantitative and qualitative nature are incurred by the classic statistical thermodynamic approaches, while PRISM theory provides a quantitative or semiquantitative, no-adjustable-parameter description of the measurements as $R_{g} / R$ was varied from 0.026 to 1.4 [33].

The wide-angle scattering peak intensity $S_{c c}\left(q_{p}\right)$ plays the role of a local collective order parameter, and displays rich and subtle trends as a function of polymer concentration and mixture size asymmetry (see figure 9). There are at least two distinct physical processes. At high colloid packing fraction, the local order is initially significantly depressed upon polymer addition if the coils are small compared to the particle. In this regime the polymers are readily dissolvable in the colloidal fluid, and the short-range depletion attraction makes the colloids 'sticky' which decreases the coherence of the local cage making it more heterogeneous. However, this trend dramatically reverses at high polymer concentrations as phase separation is approached. Here the classic depletion effect corresponding to local clustering and densification of the colloids comes into play as a precursor to phase separation, resulting in enhancement of the local order parameter. With increasing relative polymer size, the initial 'cage-melting' process becomes inefficient due to the longer range of the effective depletion attraction, and only the latter process is present. In the nanoparticle limit, very little structural modification is observed, consistent with van der Waals and WCA ideas. A similar sequence of behaviours is observed at the lower colloid packing fraction in figure 9, but the 'cage-melting' process is considerably less efficient as expected.

Changes of the local $S_{c c}(q)$ and $g_{c c}(r)$ upon polymer additions can have major dynamical consequences, which in the colloidal regime far from a glass transition can be estimated from 


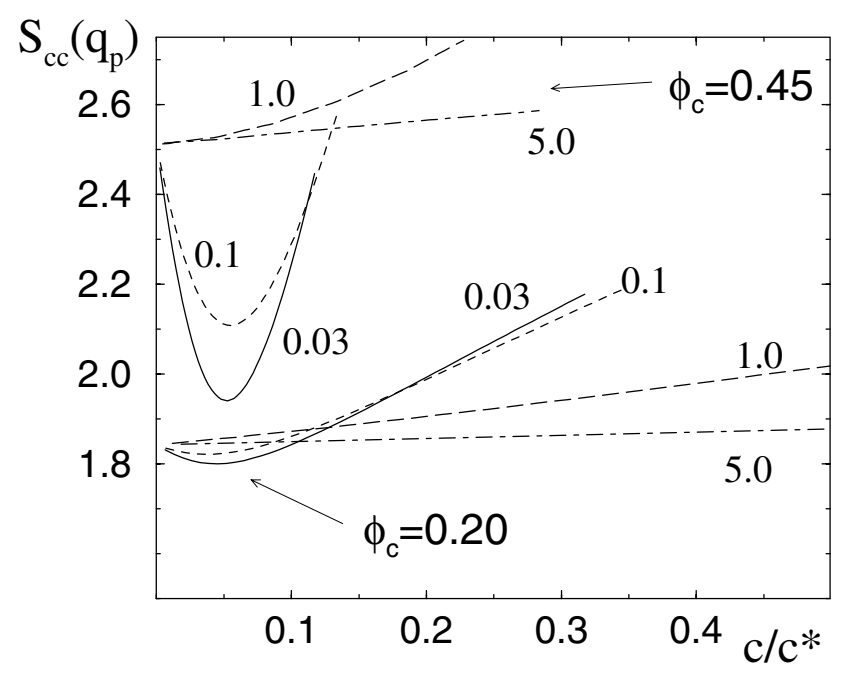

Figure 9. Large-angle scattering peak intensity, $S_{c c}\left(q_{p}\right)$, versus polymer concentration for two packing fractions, $\phi_{c}=0.45$ and 0.20 , and for the labelled polymer-colloid size ratios, $R_{g} / R$. The curves at $\phi_{c}=0.20$ are shifted upwards by 0.6 .

various statistical dynamical approaches of the generalized Enskog type [38]. With increasing colloid volume fraction, a well-studied hard-sphere glass transition occurs in the absence of polymers at $\phi_{c} \approx 0.57$. The microscopic idealized mode-coupling theory (MCT) has been successfully applied to explain this phenomenon [35]. For pure hard spheres, the essential idea is that a density is reached where the local cage constraints, quantified by the wide-angle $S_{c c}(q)$, become sufficiently strong that particles become localized on a Lindemann length scale of about $0.1 \sigma_{c}$. This is predicted to occur at a $\phi_{c} \approx 0.516$ and $S_{c c}\left(q_{p}\right) \approx 3.54$. A simple, but reliable, approximate implementation of the ideal MCT is to use the latter condition on the local order parameter as the defining criterion for glass formation. If $R_{g} / R \ll 1$, then using an effective one-component model to treat the dynamics seems sensible [35-37]. Hence, the influence of polymer additives on the value of $\phi_{c}$ at the colloidal glass transition can be predicted using the PRISM structural information.

Sample results are shown in figure 10. One sees that remarkably small concentrations of polymer can at high density significantly reduce local cage ordering, resulting in major increases of the glass transition volume fraction which nearly follow a linear dependence with $c / c^{*}$. A shift of up to $10 \%$ is experimentally relevant before the random-close-packing constraint is encountered. The polymers can effectively 'melt' a glass, and this phenomenon has been experimentally observed in model hard-sphere colloid-polymer mixtures [8]. As $R_{g} / R$ increases, the ability of polymers to disrupt the local hard-sphere packing is reduced, and the glass-melting mechanism is less effective. For $R_{g}=R$ (not shown), there is essentially no perturbation of the glass volume fraction. With increasing $c / c^{*}$, the lines in figure 10 'turn around' well before the fluid-fluid spinodal boundary is encountered, and the glass suppression effect is reversed. This curious non-monotonic trend is expected from the shape of $S_{c c}\left(q_{p}\right)$ at high colloid density shown in figure 9. We do not show this behaviour here, since it is now established within MCT that with increasing attraction strength a new non-ergodicity transition emerges that has a gelation-type character with particles localized on the length scale of the attractive interaction $\left(R_{g}\right)$ and not $\sigma_{c}$ [36,37]. Such a system is sometimes called an 'attraction-driven' glass in contrast to the classic jamming effect in hard-sphere colloids 


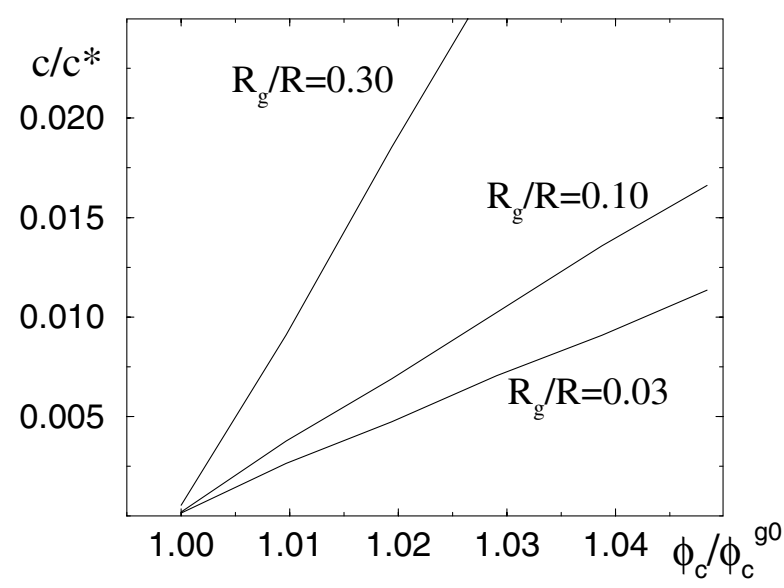

Figure 10. The shift of the glass transition line, relative to the value for hard spheres, $\phi_{c}^{g 0}=$ $\phi_{c}^{g}\left(c / c^{*}=0\right)=0.516$ (theory) or $\phi_{c}^{g 0}=0.58$ (experiment), as estimated from $S_{c c}\left(q_{p}\right)=3.54$ versus polymer concentration for size ratios as labelled.

driven by purely repulsive packing considerations, and cannot be predicted on the basis of the simple $S_{c c}\left(q_{p}\right)=3.54$ criterion valid for (nearly) hard-sphere fluids and weakly attractive colloids $[36,37]$.

\section{Polymer-colloid correlations}

In this section we investigate the influence of simultaneous non-zero polymer and colloid concentrations on the polymer segment-colloid pair correlations. Figure 11 presents representative results for a high colloid volume fraction and three values of $R_{g} / R$. The limiting cases of zero polymer concentration, and zero colloid volume fraction, are shown for comparison. The most striking result is how weakly dependent the packing correlations are on polymer concentration, especially in the more local depletion hole regime where segments are within a distance $R_{g}$ of the particle surface. This arises because the polymer coils are rather compressible, at the densities considered, below the semidilute threshold concentration, $c^{*}$. The peak in $g_{p c}(r)$ on macromolecular length scales $\left(r-R>R_{g}\right)$ is suppressed as polymer concentration increases, and more so as the colloid size increases. A physical interpretation of this trend is that as $c / c^{*}$ increases the individual identity of spherical polymeric coils is reduced due to (weak) interpenetration of different chains. The situation may be akin to that of polyelectrolyte solutions which at low salt concentration pack in a simple-liquid-like manner, with this effect gradually disappearing as the polymer concentration and/or Debye-Huckel screening length decrease [65]. However, the physics of inhomogeneity suppression in the neutral polymer-colloid mixture may be more subtle.

The inset in figure 11 shows the correlations for non-zero polymer concentration on large scales. Separation is now normalized by colloid diameter, which highlights the oscillatory correlations due to imprinting of colloid structural order on segmental packing. The weakening of the oscillatory features with increasing polymer size is intuitively expected and is also evident in the dilute-polymer limit.

Figure 12 presents analogous results for a lower colloid volume fraction where the pure hard spheres have very little solvation shell structure. Our conclusions regarding the role of non-zero polymer concentration are qualitatively the same as for figure 11: the width of the local 


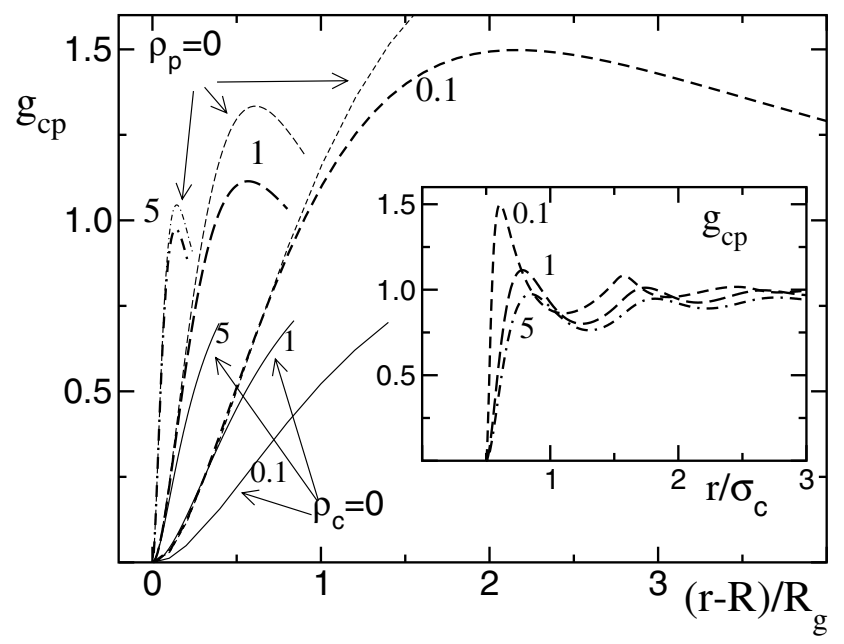

Figure 11. The profile of polymer segment density, $g_{c p}$, versus rescaled distance, $(r-R) / R_{g}$, from a particle surface; size ratios: $R_{g} / R=0.1$ (short dashes), 1.0 (long dashes) and 5.0 (dot-dashed) as labelled. As in figure 7 , the colloid concentration $\phi_{c}=0.45$, and the polymer concentration relative to the spinodal is constant, $c / c_{s}=0.8$. Thin curves of the same styles give the results for the same $R_{g} / R$ and $\phi_{c}$ but at vanishing polymer concentrations, $c / c_{s}=0$. Thin solid curves show the depletion layer for identical polymer concentrations, $c / c_{s}=0.8$, but at vanishing colloid concentration, $\phi_{c} \rightarrow 0$, at the size ratios studied, as labelled. The inset shows the finite-density curves versus $r / \sigma_{c}$ out to larger distances.

depletion layer is dominated by the colloidal concentration fluctuation length scale. However, slight narrowing of the local depletion hole is now observable, and more so as polymer size shrinks, due to weak polymer mesh formation. On macromolecular distances, suppression of the (now weak) layering behaviour is again observed, and more so as the colloids increase in relative size.

The inset of figure 12 shows the surface-area-weighted correlated part of $g_{p c}(r)$ as a function of separation normalized now by the colloid diameter. This plot again emphasizes the imprinting of oscillatory colloidal packing on segmental organization. However, it also demonstrates that there is still a long-range (power-law-tail) depletion layer component which suppresses segment-colloid contacts and is not screened away. The oscillatory features effectively 'ride' this long-range depletion tail. As a subtle consequence of the latter, even though the local depletion layer is relatively narrow on the $\sigma_{c}$-scale for $\phi_{c}=0.45, c / c_{s}=0.8$, and $R_{g} / R=5$ (see the inset of figure 11), the corresponding $g_{c c}(r)$ in figure 7 looks as one would expect for a weak, long-range attraction. This again emphasizes the existence of a long-range tail in $g_{p c}(r)$ connected to the $R_{g}$-scale. The predicted scaling law in the limit of $R_{g} \gg R$ again bears this out, as it gives for the large-distance behaviour of the depletion layer

$$
g_{c p}(r \gg R) \rightarrow 1-\frac{R}{r} A\left(\phi_{c}\right) \mathrm{e}^{-r / \xi_{c}} \quad \text { for } R_{g} \gg R,
$$

where the parameters are defined in equations (14) and (15). Thus, the depletion layer exhibits a power-law tail extending out to the collective correlation length $\xi_{c}$, which increases upon approaching phase separation as seen in the inset of figure 12. As argued below (15), we expect this result to be qualitatively valid as long as the polymer mesh size is larger than the particle, $\xi>R$.

The local width of the polymer depletion layer at non-zero polymer concentrations is shown in figure 13, and can be contrasted with its dilute-limit analogue of figure 5 . For low 


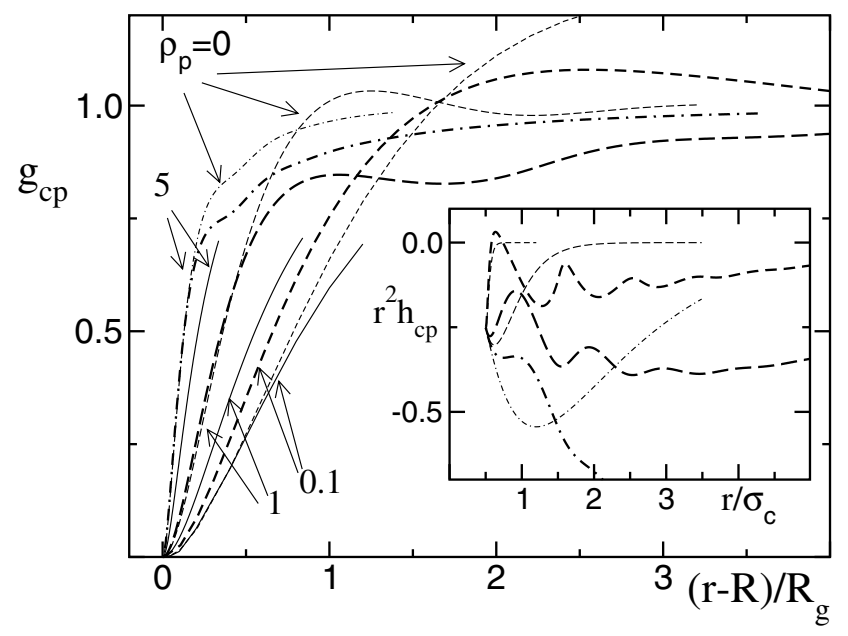

Figure 12. The main panel shows profiles of polymer segment density, $g_{c p}$, versus rescaled distance, $(r-R) / R_{g}$, as in figure 11 but at colloid concentration $\phi_{c}=0.20$; size ratios: as labelled; and $c / c_{s}=0.8(0)$ thick curves (thin curves). Thin solid curves give the results at these polymer concentrations but at $\phi_{c}=0$. The inset shows the curves at $c / c_{s}=0.8$ and 0 in the form $r^{2} h_{c p}(r)$ versus $r / \sigma_{c}$ in order to exhibit the long-range behaviour.

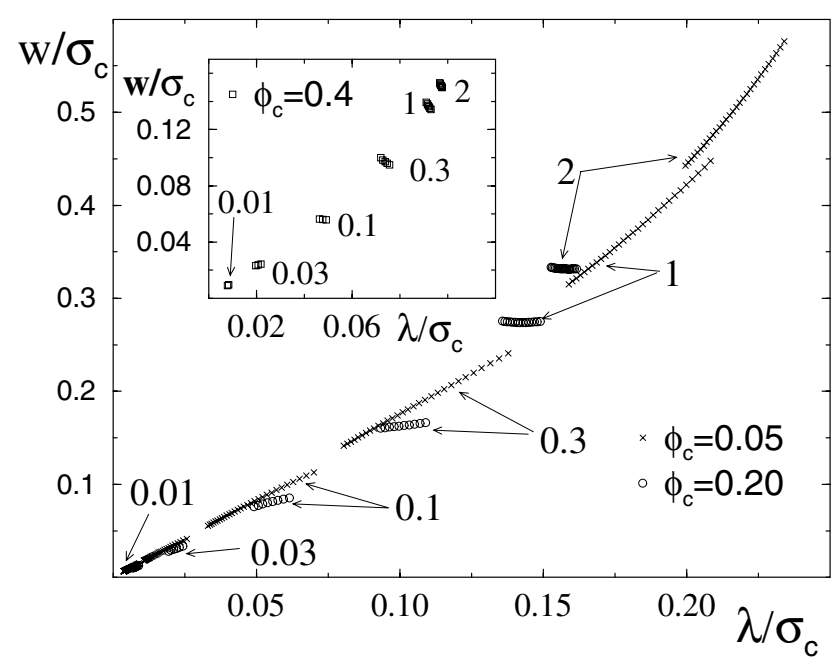

Figure 13. Local width of the polymer segment depletion layer as in figure 5 versus $\lambda$ for two colloid concentrations, $\phi_{c}=0.05$ and 0.20 , and at finite polymer concentration. The curves are labelled with the size ratio $R_{g} /\left(\sqrt{2} \sigma_{c}\right)$, and the polymer concentration increases up to $c / c^{*}=1.5$ for $\phi_{c}=0.05$ and up to the spinodals for $\phi_{c}=0.20$, respectively. $\lambda$ decreases with $c / c^{*}$. The inset shows equivalent results for $\phi_{c}=0.40$.

$\phi_{c}$, or for small polymer sizes such that $w \approx \xi$, the depletion width $w$ correlates strongly with $\lambda$ from equation (7), the length scale over which the polymer segments rearrange. For the example of $\phi_{c}=0.05$ in figure 13, both lengths decrease with increasing $c / c^{*}$ and increase with $R_{g} / R$, yet saturating at fractions of $R$ for $R_{g} / R \gg 1$. The primary finding for slightly higher colloid concentrations and/or polymer sizes such that $\xi \gg w$ is the relatively small influence of non-zero polymer concentration on the local depletion layer width. The harsh 


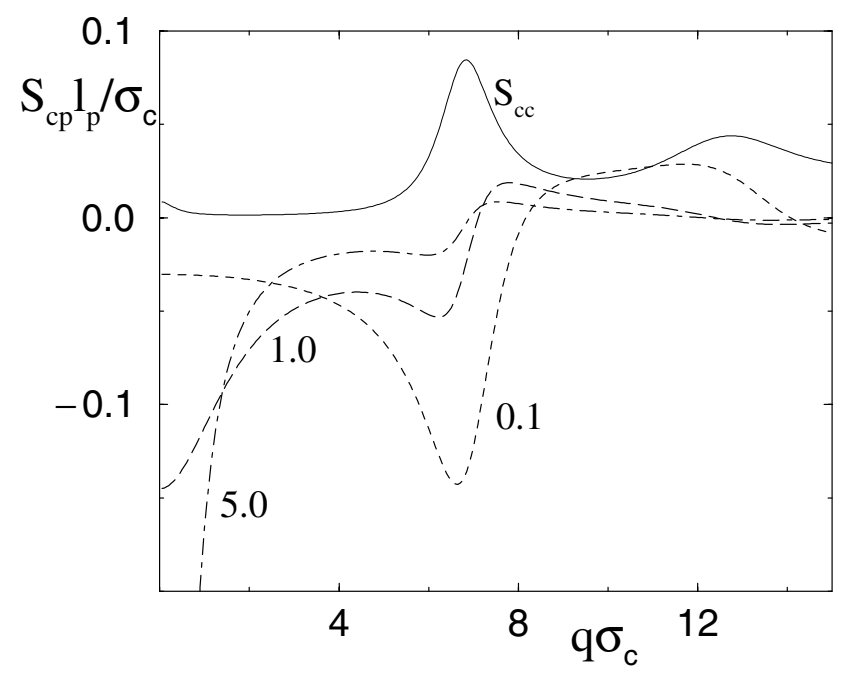

Figure 14. Polymer-colloid (cross-term) partial structure factors, $\left(l_{p} / \sigma_{c}\right) S_{c p}(q)$, at colloid concentration $\phi_{c}=0.45$ and (large) constant distance to the spinodal, $c / c_{s}=0.3$, for the three size ratios $R_{g} / R=0.1,1$, and 5 as labelled. The thin solid curve gives the corresponding (scaled) colloid structure factor $\frac{1}{30} S_{c c}(q)$ for $R_{g} / R=5$.

repulsive particle packing constraints overcome the considerations of polymer conformational entropy and the depletion layer width $w$ becomes much smaller and almost independent of $c / c^{*}$, while the non-locality length decreases with the polymer correlation length. While the case $\phi_{c}=0.4$ in figure 13 only shows this behaviour, for $\phi_{c}=0.2$ a crossover between the two trends occurs when the depletion layer becomes of the order of the relevant polymer length. Clearly, predicting the depletion layer width at finite concentrations becomes a difficult quantitative problem in general, and the accuracy of our PRISM m-PY results needs to be tested.

The Fourier space consequences of polymer-colloid packing correlations are probed via $S_{c p}(q)$, an example of which is shown in figure 14. The corresponding colloidal structure factor for the largest-polymer case is also shown. There are three distinct features. At small wavevectors, a peak is observed for the two larger-polymer cases associated with the development of long-wavelength concentration fluctuations which favour polymer-colloid demixing. It is also present in the colloidal scattering function and is a precursor to phase separation since the correlation length associated with it is $\approx 2.5 \sigma_{c}$ which is larger than even the dilute-polymer-solution correlation length. On local particle packing length scales, $q \sigma_{c} \approx 7$, a negative, liquid-like packing feature is observed associated with anti-correlation of polymer and particle positions on the colloidal first-neighbour-shell scale. Its amplitude increases as the polymers get smaller in accord with the reasoning leading to (13) for $c \ll c^{*}$. On even smaller (larger) length (wavevector) scales, $S_{c p}(q)$ changes sign, implying that positive excess polymer and colloid density are now favoured.

\section{Polymer-polymer correlations}

\subsection{Dilute polymers}

Examples of the interchain site-site correlations in the dilute limit are shown in figure 15. The molecular polymer-polymer second virial coefficient is proportional to the integral of 


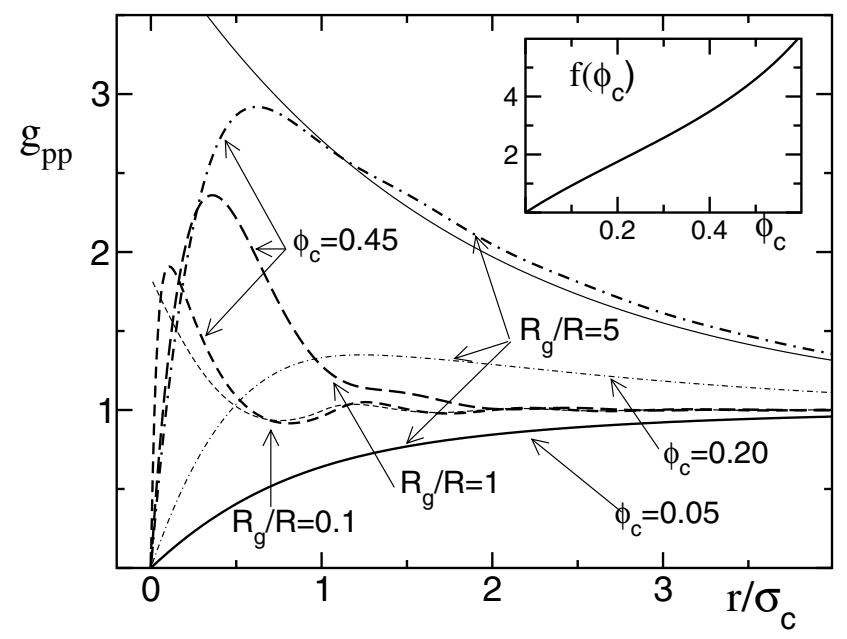

Figure 15. The polymer segment-polymer segment pair correlation function, $g_{p p}(r)$, for dilute polymers immersed in a hard-sphere solution; the colloid packing fractions $\phi_{c}$ and size ratios $\xi_{0}$ are the same as in figure 4 , as labelled. The thin solid curve presents the asymptote for large polymers, equation (18), evaluated for $R_{g} / R=5$ and $\phi_{c}=0.45$, while the thin dashed curve gives equation (17) at this $\phi_{c}$ which holds for $R_{g} \rightarrow 0$. The inset shows the intermolecular polymer segment contact value $f\left(\phi_{c}\right)$, which determines the former asymptote.

$\left(1-g_{p p}(r)\right)$. In the absence of colloids, PRISM theory properly recovers the classic $g_{p p}(r)<1$ correlation hole behaviour characterized by the length scale $\xi_{0}$ that is indicative of the "softsphere' character of the polymer coils. This correlation hole is overcome at a distance related to the depletion layer of $g_{p c}(r)$ as seen in figure 4. With increasing colloid volume fraction and/or relative polymer size, $g_{p p}(r)$ becomes increasingly positive, corresponding to a colloidmediated attractive polymer-polymer second virial coefficient [17]. A peak develops at finite $r$, and a mostly structureless decay to the random value of unity occurs with a characteristic correlation length which increases with $R_{g}$.

For small polymers at high particle density, the segmental packing displays an oscillatory behaviour due to efficient imprinting of colloidal structure on the polymer organization; see the discussion below (13). The polymer coils can explore the free volume homogeneously and thus their correlations are equivalent to those of the particles, $g^{\mathrm{HS}}$, convoluted with their (fixed) internal density correlations, $P(r)=\left(r-\sigma_{c}\right)^{2}\left(\sigma_{c}+r / 2\right) \Theta\left(\sigma_{c}-r\right)$ whose Fourier transform is proportional to the particle form factor $P(q)$ :

$g_{p p}(r)=1+\frac{\pi \varrho_{c} / 6}{\left(1-\phi_{c}\right)^{2}}\left[P(r)+\varrho_{c} \int \mathrm{d}^{3} s P(r-s)\left(g^{\mathrm{HS}}(s)-1\right)\right] \quad$ for $R_{g} \rightarrow 0$.

This limiting result is included in figure 15 and explains the higher (than that corresponding to the average density $\varrho_{p}$ ) probability for two small coils to be close at small distances.

In the $R_{g} / R \gg 1$ nanoparticle limit, an asymptotic analytic expression can be derived for $g_{p p}(r)[17]:$

$$
g_{p p}(r) \rightarrow 1+\left(f\left(\phi_{c}\right)-1\right) \mathrm{e}^{-r / \xi_{0}},
$$

where $f\left(\phi_{c}\right)$ from equation (15) is shown in the inset of figure 15. The transition from a depletion hole to enhanced packing ( $g_{p p}(r)$ approaches unity from above) occurs at $\phi_{c}=0.11$. For $\phi_{c}=0.5$, the density of segments on the second chain within a distance of $R_{g}$ from a tagged segment on the first chain is more than an order of magnitude larger than in the absence 


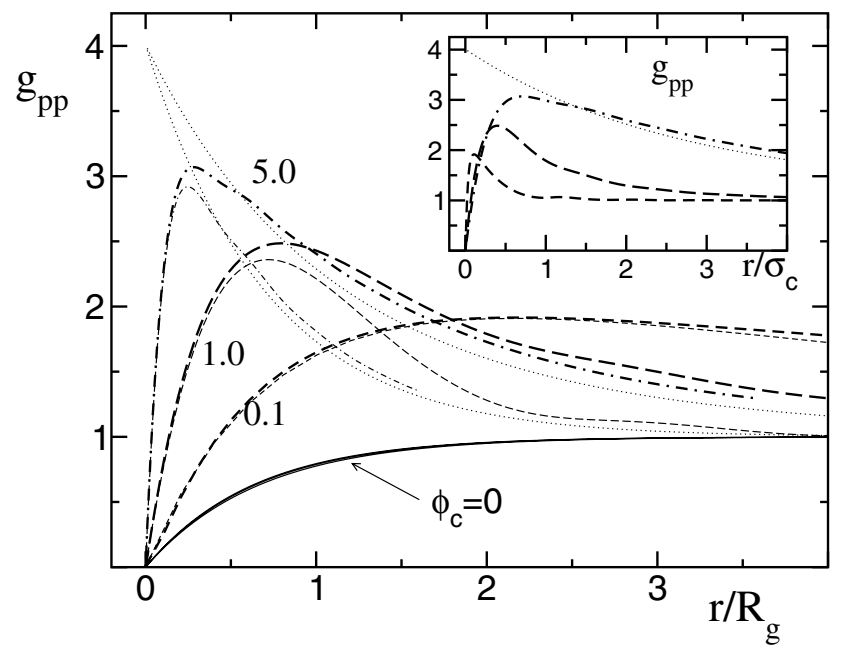

Figure 16. Polymer segment-polymer segment pair correlation functions, $g_{p p}(r)$, versus radial distance in units of $R_{g}$ for the size ratios $R_{g} / R=0.1$ (short dashes), 1.0 (long dashes), and 5.0 (dot-dashed). As in figure 11, the colloid concentration is $\phi_{c}=0.45$, and the polymer concentration relative to the spinodal is constant, $c / c_{s}=0.8$ (bold) and 0 (thin). The thin solid (almost overlapping) curves give the results for $c / c_{s}=0.8$ and vanishing colloid concentrations, $\phi_{c}=0$. Dotted curves indicate the asymptote for $R_{g} / R \rightarrow \infty$ evaluated for the two curves at $R_{g} / R=5$. The inset shows the finite-density results (thick lines) and the large- $R_{g}$ asymptote from the main panel as a function of $r / \sigma_{c}$ (thin dashes).

of colloids. The colloid-induced enhancement of polymer-polymer contacts is a phenomenon not treated by effective potential models. It depends systematically on $R_{g} / R$ and $\phi_{c}$, and implies a tendency for correlated polymer clustering. The latter has strong thermodynamic consequences, and plays an essential role in the novel predictions of PRISM theory for the fluid-fluid demixing transition $[16,17,32]$.

\subsection{Concentrated polymers}

Examples of the influence of non-zero polymer concentration on polymer segment-segment pair correlations are given in figure 16 for a high colloid volume fraction. The analogous results for vanishing polymer or colloid concentration are also presented for comparison. As found for the polymer-colloid pair correlations, the most striking aspect of figure 16 is the relative insensitivity of the segmental correlations to polymer concentration at the rather low values of $c / c^{*}$ which characterize the homogeneous one-phase regime. For $r \ll R_{g}$, a correlation hole is present which is only slightly narrower than in the $c \rightarrow 0$, dilute-polymer limit. The hole caused by the mutual soft repulsion of the coils crosses over to an enhanced segmental packing correlation $\left(g_{p p}>1\right)$ on a length scale comparable to $R_{g}\left(\sigma_{c}\right)$ for small (large) polymer radii. Small increases of intensity and location of the latter feature occur at non-zero polymer concentration, although the distance of decay to the ultimate random value of unity is clearly enhanced due to pre-transitional long-wavelength composition fluctuations. The $R_{g} \gg R$ scaling law corresponding to (14) and (16) is given by

$$
g_{p p}(r) \rightarrow 1+\frac{1}{b} \frac{R}{r}\left(\mathrm{e}^{-r / \xi_{c}}-\mathrm{e}^{-r / \xi_{0}}\right) \quad \text { for } R_{g} \gg R
$$

This result demonstrates that long-wavelength correlations can have an amplitude proportional to the inverse of the small parameter, $b \sim\left(c / c^{*}\right)\left(R / R_{g}\right)$, which quantifies the fraction of 


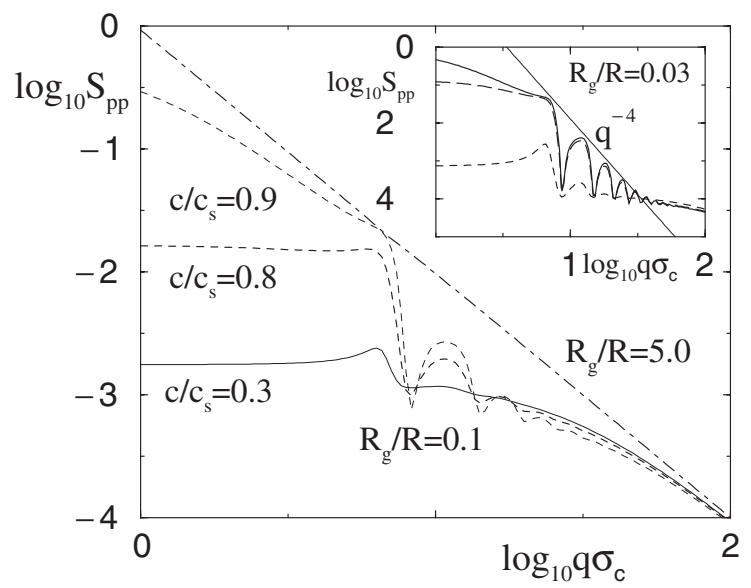

Figure 17. A double-logarithmic plot of the collective polymer structure factor, $S_{p p}(q)$ in units of $\left(\sigma_{c} / l_{p}\right)^{2}$, for colloid packing fraction $\phi_{c}=0.45$, constant distance to the spinodal, $c / c_{s}=0.8$ (thick curves), and two size ratios, $R_{g} / R=0.1$ (dashed) and 5 (dash-dotted), as labelled. For the smaller polymer, the curves for $c / c_{s}=0.3$ (thin solid) and $c / c_{s}=0.9$ (thin dashes) are also shown. The inset shows $S_{p p}$ for size ratio $R_{g} / R=0.03$ and the polymer concentrations $c / c_{s}=0.3$ (short dashes), 0.6 (long dashes), and 0.8 (solid), while a thin solid curve corresponds to the Porod-scattering law $q^{-4}$.

displaced segments. The dotted lines show that the limit of asymptotically large polymer is rather closely approached already when $R_{g} / R=5$, and that adding polymer dominantly increases the collective correlation length, $\xi_{c}$ of (15), because it drives the system closer to the spinodal demixing instability.

The inset of figure 16 shows the non-zero polymer concentration results on an expanded scale with intersegment separation non-dimensionalized by colloid diameter. A weak oscillatory feature is present for the smallest polymer, indicating that they again fill the voids between the particles rather homogeneously, so (17) could be generalizable to finite densities.

An example of the polymer-polymer structure factor is given in figure 17 in a log-log format. Results are shown for a high colloid volume fraction and a large and a small value of $R_{g} / R$. For the larger-polymer case, and even if $R_{g}=R$ (not shown), the scattering function is very nearly a simple Lorentzian: $S_{p p}(q)=\left(\xi_{c} / l_{p}\right)^{2} /\left(1+\left(q \xi_{c}\right)^{2}\right)$, as follows from the large- $R_{g}$ asymptotic behaviour, equation (19). However, for small enough polymers such as the $R_{g} / R=0.1$ case shown, the polymer scattering pattern displays distinctive regimes as a function of polymer concentration and wavevector. For $q R_{g}>1$, intramolecular single-chain correlations are probed and the Gaussian chain power law $q^{-2}$ is found. For $q R_{g}<1$, but $q \sigma_{c}>7$, oscillatory features emerge with increasing polymer concentration. As discussed in section 3.2, this behaviour is a consequence of the simple fact that polymer density must vanish where the colloids are, and that in the free-volume space, where the polymers are located, they are distributed in an almost homogeneous manner on scales larger than the radius of gyration. Therefore, the relative amplitude, and maxima and minima, should be well described by the classic form factor of a homogeneous sphere: $P(q)=\left[3 /(q R)^{4}\right][\cos q R-\sin q R / q R]^{2}$. This is indeed what we find, and the classic Porod law $q^{-4}$ appropriate for scattering from a sharp interface between two phases is observed with increasing polymer concentration, and at smaller values of $c / c_{s}$ as $R / R_{g}$ increases (see the inset). 
For other situations and parameter values we find (not shown) behaviour similar to that shown in figure 17. For example, at a relatively high fixed value of $c / c^{*}$ for $R_{g} \ll R$, a very similar sequence of scattering profiles is found as the spinodal is approached on increasing $\phi_{c}$.

\section{Discussion}

We close with a brief discussion of the limitations of the present version of PRISM/m-PY theory, and future opportunities for generalization and applications.

For the athermal polymer-particle mixture problem, there are at present five primary limitations that we can identify.

(1) Use of the PY closure for colloid-colloid direct correlations can break down when the depletion attraction becomes much stronger than the thermal energy $k T[16,51,58]$. This is most relevant when $R \gg R_{g}$ and $\phi_{c}$ is small, and may play an important role in the prediction of complete miscibility (in the spinodal sense) at small colloid volume fractions, seen in figure 6.

(2) The thread model is not appropriate at high (melt-like) polymer densities, nor for persistent chains mixed with relatively small particles, situations for which the specific monomer size and/or polymer backbone stiffness become relevant.

(3) The use of a Gaussian polymer structure factor for athermal solution conditions instead of the more open self-avoiding-walk model is expected to overestimate depletion effects, and more so as $R_{g} / R$ increases.

(4) A fully self-consistent treatment of single-chain correlations which accounts for both screening of intramolecular excluded-volume interactions by polymer concentration fluctuations [66], and possible colloid-induced conformational changes, has not been carried out. The latter effect is expected to become more important as $R_{g} / R$ increases and polymers must 'wrap around' the particles.

(5) Our results for fluid-fluid phase separation are currently at the spinodal instability (plus critical point) level; numerical construction of full binodal coexistence curves remains to be achieved. Work is in progress on this.

Both new experiments and computer simulations would be very valuable for testing our predictions. Small-angle and wide-angle scattering experiments to extract the colloid-colloid, and polymer-polymer, structure factors can be directly compared with the theoretical results in wavevector space. Alternatively, in the colloidal regime, real-space video microscopy measurements could test our radial distribution predictions for $g_{c c}(r)$. Systematic phase diagram studies in the true nanoparticle regime over a wide range of particle volume fractions could further test our novel predictions for fluid-fluid phase separation and the location of critical points. Computer simulations which include polymer-polymer repulsive forces should be feasible in the nanoparticle regime, and would provide detailed information concerning both structure and miscibility. Simulations of the elementary one- and two-particle (or polymer) problems appear to not be available and would be the best model systems to investigate first and compare with theory.

As regards future extensions of the integral equation theory, there are multiple possibilities which present variable degrees of technical and conceptual challenge.

First, either analytically, or with modest numerical effort, our results for one and two particles in a polymer solution can be generalized to non-Gaussian coil architectures characterized by arbitrary fractal dimensions $[47,48]$. This is especially relevant to experiments which probe the second virial coefficient of nanoparticles (e.g., proteins [57]) which are sensitive to polymer conformational statistics [27]. 
Second, at non-zero concentrations of both species our use of the Baxter factorization method to (nearly) solve the coupled integral equations is no longer applicable for polymers that are not described by a Gaussian structure factor. Hence, a fully numerical approach must be employed. This will be most tractable within the 'thread' level description where the polymer-monomer excluded-volume diameter is shrunk to zero. Variable polymer structures (e.g., rods, stars) can be modelled by adopting the appropriate single-macromolecule structure factor. Semiflexible chains can be modelled by introducing the chain persistence length scale, and the crossover of $\omega(q)$ from rigid-rod-like to random-coil-like with decreasing wavevector. Modification of the detailed form of the m-PY closure is expected to be required to properly reflect polymer conformational statistics on length scales smaller than the non-locality length $\lambda$. Based on a numerical approach, generalization to treat non-spherical particles, or spheres with heterogeneous surfaces [67], is natural within the site representation of RISM theory as long as strong orientational correlations and liquid-crystal formation are not relevant [21].

In principle, one can address the role of non-hard-core forces, such as van der Waals attractions or Coulomb interactions [68], between polymer and/or colloidal species, including the question of variable solvent quality for the polymer chain. However, this can be a difficult task within integral equation theory when such variable strength and spatial range interactions can induce significant changes in mixture structure. For example, if strong attractions (or noncontact repulsions) exist between polymer segments and particles, then collective phenomena akin to wetting/adsorption (dewetting/drying) can occur which are generally not properly captured with standard closure approximations [69]. However, the availability of field theoretic results for the simplest realization of such problems [6,27-29] may again provide critical guidance for the development of new closure approximations. The question of conformational perturbations of the polymer may also become more important, especially if the polymer experiences strong attractions to the colloids or nanoparticles. Application of existing selfconsistent schemes $[6,26,66,70]$, including hybrid PRISM plus field theoretic or Monte Carlo simulation approaches which have successfully treated intrapolymer excluded volume [66], are then required. Of course, the treatment of Coulomb forces remains a major challenge even for spherical colloids [68], although progress has been recently made within the integral equation framework for charged spheres and/or polyelectrolytes [66,71].

Finally, high-density macromolecular systems are relevant to materials such as (nano-) particle-filled polymer melts. Here a thread description is not appropriate, but the basic m-PY idea should again be applicable to account for the local (chemically specific) perturbation of polymer chains near a particle surface. An explicit treatment of the non-zero monomer diameter and other local chain structural features is required, which should be numerically feasible if the particles are of nanoscopic dimensions.

\section{Acknowledgments}

We acknowledge helpful discussions with L Belloni, A P Chatterjee, Y L Chen, S Egelhaaf, E Eisenriegler, A Johner, A Moussaid, W Poon, P Pusey, M Schmidt and C Zukoski. MF was supported by the Deutsche Forschungsgemeinschaft under grant no Fu 309/3 and through the SFB 563. KSS was supported by the US Department of Energy grant number DEFG0291ER45439 through the UIUC Materials Research Laboratory.

\section{References}

[1] Napper D H 1983 Polymeric Stabilization of Colloidal Dispersions (New York: Academic)

[2] Vincent B 1974 Adv. Colloid Interface Sci. 4193 
[3] Russel W B, Saville D A and Schowalter W R 1989 Colloidal Dispersions (Cambridge: Cambridge University Press)

[4] Enikolopyan N S, Fridman M L, Stalnova I O and Popov V L 1990 Adv. Polym. Sci. 961

[5] Lewis J A 2000 J. Am. Ceram. Soc. 832341

[6] de Gennes P G 1979 Scaling Concepts in Polymer Physics (Ithaca, NY: Cornell University Press) des Cloizeaux J and Jannik G 1990 Polymers in Solution: Their Modelling and Structure (Oxford: Oxford University Press)

Schäfer L 1999 Excluded Volume Effects in Polymer Solutions (Berlin: Springer)

[7] Moussaid A, Poon W, Pusey P N and Soliva M F 1999 Phys. Rev. Lett. 82225

[8] Ilett S M, Orrock A, Poon W C K and Pusey P N 1995 Phys. Rev. E 511344

[9] Poon W C K, Starrs L, Meeker S P, Moussaid A, Evans R M L, Pusey P N and Robins M M 1999 Faraday Discuss. 112143

[10] Segre P N, Prasad V, Schofield A B and Weitz D A 2001 Phys. Rev. Lett. 866042

[11] Robb I D, Williams P A, Warren P B and Tanaka R 1995 J. Chem. Soc. Faraday Trans. 913901 Clegg S M, Williams P, Warren P and Robb I D 1994 Langmuir 103390

[12] Ye X, Narayanan T, Tong P, Huang J S, Lin M Y, Carvalho and Fetters L J 1996 Phys. Rev. E 546500

[13] Tuinier R, Dhont J K G and deKruif C G 2000 Langmuir 161497

[14] Verhaegh N A M, van Duijneveldt J S, Dhont J K G and Lekkerkerker H N W 1996 Physica A 230409

[15] Schaink H M and Smit J A M 1997 J. Chem. Phys. 1071004

[16] Fuchs M and Schweizer K S 2000 Europhys. Lett. 51621

[17] Fuchs M and Schweizer K S 2001 Phys. Rev. E 64021514

[18] Schweizer K S and Curro J G 1987 Phys. Rev. Lett. 58246

[19] Schweizer K S and Curro J G 1994 Adv. Polym. Sci. 116319 Schweizer K S and Curro J G 1997 Adv. Chem. Phys. 981

[20] Chandler D and Andersen H C 1972 J. Chem. Phys. 571930

[21] Chandler D 1982 Studies in Statistical Mechanics vol 8, ed E W Montroll and J L Lebowitz (Amsterdam: North-Holland) p 274

[22] Chandler D 1993 Phys. Rev. E 482898

[23] Asakura S and Oosawa F 1954 J. Chem. Phys. 221255

Asakura S and Oosawa F 1958 J. Polym. Sci. 33183

Vrij A 1976 Pure Appl. Chem. 48471

[24] Gast A P, Hall C K and Russel W B 1983 J. Colloid Interface Sci. 96251

[25] Dijkstra M, Brader J M and Evans R 1999 J. Phys.: Condens. Matter 1110079 Dijkstra M, van Roij R and Evans R 1999 Phys. Rev. E 595744 Dijkstra M, van Roij R and Evans R 2000 J. Chem. Phys. 1134799

[26] Muthukumar M 1989 J. Chem. Phys. 904594 Leung K and Chandler D 1994 Phys. Rev. E 492851

[27] Eisenriegler E 2000 J. Chem. Phys. 1135091

[28] Eisenriegler E 1997 Phys. Rev. E 553116

[29] Hanke A, Eisenriegler E and Dietrich S 1999 Phys. Rev. E 596853 Eisenriegler E, Hanke A and Dietrich S 1996 Phys. Rev. E 641134

[30] Lekkerkerker H N W, Poon W C, Pusey P N, Stroobants A and Warren P B 1992 Europhys. Lett. 20559 Warren P B, Ilett S M and Poon W C K 1995 Phys. Rev. E 525205

[31] Widom B and Rowlinson J S 1970 J. Chem. Phys. 521670

[32] Ramakrishnan S, Fuchs M, Schweizer K S and Zukoski C F 2002 J. Chem. Phys. 1162201

[33] Ramakrishnan S, Fuchs M, Schweizer K S and Zukoski C F 2001 Langmuir 181082

[34] Hansen J P and McDonald I R 1986 Theory of Simple Liquids (London: Academic)

[35] Götze W and Sjögren L 1992 Rep. Prog. Phys. 55241

[36] Bergenholtz J and Fuchs M 1999 Phys. Rev. E 595706

[37] Fabbian L, Götze W, Sciortino F and Thiery P T 1999 Phys. Rev. E 59 R1347

[38] Cohen E G D, Verberg R and de Schepper I M 1998 Physica A 251251

[39] Meijer E J and Frenkel D 1991 Phys. Rev. Lett. 671110 Meijer E J and Frenkel D 1994 J. Chem. Phys. 1006873 Meijer E J and Frenkel D 1995 Physica A 213130

[40] Bolhuis P and Frenkel D 1994 J. Chem. Phys. 1019869

[41] Dickman R and Yethiraj A 1994 J. Chem. Phys. 1004683

[42] Louis A A, Finken R and Hansen J P 1999 Europhys. Lett. 46741

[43] Bolhuis P G, Louis A A, Hansen J P and Meijer E J 2001 J. Chem. Phys. 1144296 
Louis A A, Finken R and Hansen J P 2000 Phys. Rev. E 61 R1028

Louis A A 2000 Phys. Rev. Lett. 841840

Louis A A, Bolhuis P and Hansen J P 2000 Phys. Rev. E 627961

[44] Pickett G T and Schweizer K S 2000 J. Chem. Phys. 1124869

Pickett G T and Schweizer K S 2000 J. Chem. Phys. 1124881

[45] Grayce C J and Schweizer K S 1995 Macromolecules 287461

[46] Khalatur P G, Zherenkova L V and Khoklov A R 1997 J. Physique 7543

Khalatur P G, Zherenkova L V and Khoklov A R 1997 Physica A 247205

Shusharina N P, Khalatur P G and Khoklov A R 2000 J. Chem. Phys. 1137006

[47] Schweizer K S and Curro J G 1988 Macromolecules 213070

Schweizer K S and Curro J G 1988 Macromolecules 213082

Fuchs M and Schweizer K S 1997 J. Chem. Phys. 106347

[48] Fuchs M 1997 Z. Phys. B 103521

Fuchs M and Müller M 1999 Phys. Rev. E 601921

[49] Lebowitz J L and Rowlinson J S 1964 J. Chem. Phys. 41133

[50] Joanny J F, Leibler L and de Gennes P G 1979 J. Polym. Sci.: Polym. Phys. 171073

[51] Chatterjee A P and Schweizer K S 1998 J. Chem. Phys. 10910464

Chatterjee A P and Schweizer K S 1998 J. Chem. Phys. 10910477

Chatterjee A P and Schweizer K S 1999 Macromolecules 32923

[52] Krakoviack V, Hansen J P and Louis A A 2001 Preprint cond-mat/0110387

[53] Tuinier R, Vliegenthart G A and Lekkerkerker H N W 2001 J. Chem. Phys. 11310768

[54] de Gennes P G 1979 C. R. Acad. Sci., Paris B 288359

[55] Odijk T 2000 Physica A 278347

Odijk T 1996 Macromolecules 291842

[56] Sear R P 1998 Eur. J. Phys. B 1313

[57] Kulkarni A M, Chatterjee A P, Schweizer K S and Zukoski C F 1999 Phys. Rev. Lett. 834554 Kulkarni A M, Chatterjee A P, Schweizer K S and Zukoski C F 2000 J. Chem. Phys. 1139863

[58] Dickman R, Attard P and Simonian V 1997 J. Chem. Phys. 107205 Attard P and Patey G N 1990 J. Chem. Phys. 924970

[59] Schmidt M, Löwen H, Brader J M and Evans R 2000 Phys. Rev. Lett. 851934 Schmidt M 2000 Phys. Rev. E 623799

[60] Baxter R J 1968 J. Chem. Phys. 492770

[61] Heyes D M and Aston P J 1992 J. Chem. Phys. 975738 Hagen M H J and Frenkel D 1994 J. Chem. Phys. 1014093

[62] Sear R 2001 Phys. Rev. Lett. 864696

[63] Chandler D, Weeks J D and Andersen H C 1983 Science 220787 Widom B 1967 Science 157375

[64] Seaton N A and Glandt E D 1987 J. Chem. Phys. 871785 Kranendonk W G T and Frenkel D 1988 Mol. Phys. 64403

[65] Yethiraj A 1998 J. Chem. Phys. 1081184

[66] Grayce C J and Schweizer K S 1994 J. Chem. Phys. 1006846 Melenkevitz J M, Schweizer K S and Curro J G 1993 J. Chem. Phys. 995571 Grayce C J, Yethiraj A and Schweizer K S 1994 J. Chem. Phys. 1006857

[67] Rosenbaum D, Zamora P C and Zukoski C F 1996 Phys. Rev. Lett. 76150 Asherie N, Lomakin A and Benedek G B 1996 Phys. Rev. Lett. 779832 Sear R P 1999 J. Chem. Phys. 1114800

[68] Belloni L 2000 J. Phys.: Condens. Matter 12 R549 Hansen J P and Löwen H 2000 Annu. Rev. Phys. Chem. 51209

[69] Evans R 1992 Fundamentals of Inhomogeneous Fluids ed D Henderson (New York: Dekker) Lum K, Chandler D and Weeks J D 1999 J. Phys. Chem. B 1034570

[70] Haronska P and Vilgis T A 1997 J. Chem. Phys. 1077502 Shaw M R and Thirumalai D 1991 Phys. Rev. A 44 R4797 van der Shoot P 1998 Macromolecules 314635

[71] Ferreira P G, Dymitrowska M and Belloni L 2000 J. Chem. Phys. 1139849 Mendez-Alcaraz J M and Klein R 2000 Phys. Rev. E 614095 